\title{
Integrals for functions with values in a partially ordered vector space
}

\author{
A. C. M. van Rooij ${ }^{1}$ • W. B. van Zuijlen ${ }^{2}$
}

Received: 22 May 2015 / Accepted: 7 December 2015 / Published online: 30 December 2015

(C) The Author(s) 2015. This article is published with open access at Springerlink.com

\begin{abstract}
We consider integration of functions with values in a partially ordered vector space, and two notions of extension of the space of integrable functions. Applying both extensions to the space of real valued simple functions on a measure space leads to the classical space of integrable functions.
\end{abstract}

Keywords Partially ordered vector space $\cdot$ Riesz space $\cdot$ Bochner integral ·

Pettis integral · Integral · Vertical extension · Lateral extension

Mathematics Subject Classification 28B05 $\cdot$ 28B15

\section{Introduction}

For functions with values in a Banach space there exist several notions of integration. The best known are the Bochner and Pettis integrals (see [1,2]). These have been thoroughly studied, yielding a substantial theory (see Chapter III in the book by Hille and Phillips [3]).

As far as we know, there is no notion of integration for functions with values in a partially ordered vector space; not necessarily a $\sigma$-Dedekind complete Riesz space. In this paper we present such a notion. The basic idea is the following. (Here, $E$ is a partially ordered vector space in which our integrals take their values.)

$凶$ W. B. van Zuijlen

willem.van.zuijlen@math.leidenuniv.nl

1 Department of Mathematics, Radboud University Nijmegen, P.O. Box 9010, 6500 GL Nijmegen, The Netherlands

2 Mathematical Institute, Leiden University, P.O. Box 9512, 2300 RA Leiden, The Netherlands 
In the style of Daniell [4] and Bourbaki [5, Chapter 3,4], we do not start from a measure space but from a set $X$, a collection $\Gamma$ of functions $X \rightarrow E$, and a functional $\varphi: \Gamma \rightarrow E$, our "elementary integral". We describe two procedures for extending $\varphi$ to a larger class of functions $X \rightarrow E$. The first (see Sect. 3), the "vertical extension", is analogous to the usual construction of the Riemann integral, proceeding from the space of simple functions. The second (see Sect.4), the "lateral extension", is related to the improper Riemann integral.

In Sect. 5 we investigate what happens if one repeatedly applies those extension procedures, without considering the space $E$ to be $\sigma$-Dedekind complete or even Archimedean. However, under some mild conditions on $E$ one can embed $E$ into a $\sigma$-Dedekind complete space. In Sect. 6 we discuss the extensions procedures in the larger space. Sections 7 and 8 treat the situation in which $\Gamma$ consists of the simple $E$-valued functions on a measure space. (In Sect. 7 we have $E=\mathbb{R}$.) In Sect. 9 we consider connections of our extensions with the Bochner and the Pettis integrals for the case where $E$ is a Banach lattice. In Sect. 10 we apply our extensions to the Bochner integral. For more alternative approaches we refer to the discussion in Sect. 11.

\section{Some notation}

$\mathbb{N}$ is $\{1,2,3, \ldots\}$.

Let $X$ be a set. We write $\mathcal{P}(X)$ for the set of subsets of $X$. For a subset $A$ of $X$ :

$$
\mathbb{1}_{A}(x)= \begin{cases}1 & \text { if } x \in A, \\ 0 & \text { if } x \notin A\end{cases}
$$

As a shorthand notation we write $\mathbb{1}=\mathbb{1}_{X}$.

Let $E$ be a vector space. We write $x=\left(x_{1}, x_{2}, \ldots\right)$ for functions $x: \mathbb{N} \rightarrow E$ (i.e., elements of $E^{\mathbb{N}}$ ) and we define

$$
c_{00}[E]=\left\{x \in E^{\mathbb{N}}: \exists N \forall n \geq N\left[x_{n}=0\right]\right\}, \quad c_{00}=c_{00}[\mathbb{R}]
$$

We write $c_{0}$ for the set of sequences in $\mathbb{R}$ that converge to $0, c$ for the set of convergent sequences in $\mathbb{R}, \ell^{\infty}(X)$ for the set of bounded functions $X \rightarrow \mathbb{R}, \ell^{\infty}$ for $\ell^{\infty}(\mathbb{N})$, and $\ell^{1}$ for the set of absolutely summable sequences in $\mathbb{R}$. We write $e_{n}$ for the element $\mathbb{1}_{\{n\}}$ of $\mathbb{R}^{\mathbb{N}}$.

For a complete $\sigma$-finite measure space $(X, \mathcal{A}, \mu)$ we write $\mathcal{L}^{1}(\mu)$ for the space of integrable functions, $L^{1}(\mu)=\mathcal{L}^{1}(\mu) / \mathcal{N}$ where $\mathcal{N}$ denotes the space of functions that are zero $\mu$-a.e. Moreover we write $L^{\infty}(\mu)$ for the space of equivalence classes of measurable functions that are bounded almost everywhere.

For a subset $\Gamma$ of a partially ordered vector space $\Omega$, we write $\Gamma^{+}=\{f \in \Gamma: f \geq$ $0\}$. If $\Lambda, \Upsilon \subset \Omega$ and $f \leq g$ for all $f \in \Lambda$ and $g \in \Upsilon$ we write $\Lambda \leq \Upsilon$; if $\Lambda=\{f\}$ we write $f \leq \Upsilon$ instead of $\{f\} \leq \Upsilon$ etc. For a sequence $\left(h_{n}\right)_{n \in \mathbb{N}}$ in a partially ordered vector space we write $h_{n} \downarrow 0$ if $h_{1} \geq h_{2} \geq h_{3} \geq \cdots$ and $\inf _{n \in \mathbb{N}} h_{n}=0$. 


\section{The vertical extension}

Throughout this section, $E$ and $\Omega$ are partially ordered vector spaces, $\Gamma \subset \Omega$ is a linear subspace and $\varphi: \Gamma \rightarrow \mathrm{E}$ is order preserving and linear. Additional assumptions are given in 3.14 .

Definition 3.1 Define

$$
\Gamma_{v}=\left\{f \in \Omega: \sup _{\sigma \in \Gamma: \sigma \leq f} \varphi(\sigma)=\inf _{\tau \in \Gamma: \tau \geq f} \varphi(\tau)\right\},
$$

and $\varphi_{v}: \Gamma_{v} \rightarrow E$ by

$$
\varphi_{v}(f)=\sup _{\sigma \in \Gamma: \sigma \leq f} \varphi(\sigma) \quad\left(f \in \Gamma_{v}\right)
$$

Note: If $f \in \Omega$ and there exist subsets $\Lambda, \Upsilon \subset \Gamma$ with $\Lambda \leq f \leq \Upsilon$ such that $\sup \varphi(\Lambda)=\inf \varphi(\Upsilon)$, then $f \in \Gamma_{v}$ and $\varphi_{v}(f)=\inf \varphi(\Upsilon)$

3.2 The following observations are elementary.

(a) $\Gamma \subset \Gamma_{v}$ and $\varphi_{v}(\tau)=\varphi(\tau)$ for all $\tau \in \Gamma$.

(b) $\Gamma_{v}$ is a partially ordered vector space and $\varphi_{v}$ is a linear order preserving map ${ }^{1}$.

(c) $\left(\Gamma_{v}\right)_{v}=\Gamma_{v}$ and $\left(\varphi_{v}\right)_{v}=\varphi_{v}$.

(d) If $\Pi$ is a subset of $\Gamma$, then $\Pi_{v} \subset \Gamma_{v}$.

Of more importance to us than $\Gamma_{v}$ and $\varphi_{v}$ is the following variation in which we consider only countable subsets of $\Gamma$.

Definition 3.3 Let $\Gamma_{V}$ be the set consisting of those $f$ in $\Omega$ for which there exist countable sets $\Lambda, \Upsilon \subset \Gamma$ with $\Lambda \leq f \leq \Upsilon$ such that

$$
\sup \varphi(\Lambda)=\inf \varphi(\Upsilon)
$$

From the remark following Definition 3.1 it follows that $\Gamma_{V}$ is a subset of $\Gamma_{v}$ and that (for $f$ and $\Lambda$ as above) $\varphi_{v}(f)$ is equal to $\sup \varphi(\Lambda)$. We will write $\varphi_{V}=\left.\varphi_{v}\right|_{\Gamma_{V}}$. We call $\Gamma_{V}$ the vertical extension ${ }^{2}$ under $\varphi$ of $\Gamma$ and $\varphi_{V}$ the vertical extension of $\varphi$.

In what follows we will only consider $\varphi_{V}$ and not $\varphi_{v}$. However, most of the theory presented can be developed similarly for $\varphi_{v}$. (For comments see 11.2.)

Example 3.4 $\Gamma_{V}$ is the set of Riemann integrable functions on $[0,1]$ and $\varphi_{V}$ is the Riemann integral in case $E=\mathbb{R}, \Omega=\mathbb{R}^{[0,1]}$ and $\Gamma$ is the linear span of $\left\{\mathbb{1}_{I}\right.$ : $I$ is an interval in $[0,1]\}$ and $\varphi$ is the Riemann integral on $\Gamma$.

3.5 In analogy with 3.2 we have the following.

\footnotetext{
${ }^{1}$ This follows from the following fact: Let $A, B \subset E$. If $A$ and $B$ have suprema (infima) in $E$, then so does $A+B$ and $\sup (A+B)=\sup A+\sup B \quad(\inf (A+B)=\inf A+\inf B)$.

2 One could also define the vertical extension in case $E, \Omega, \Gamma \subset \Omega$ are partially ordered sets (not necessarily vector spaces) and $\varphi: \Gamma \rightarrow E$ is an order preserving map.
} 
(a) $\Gamma \subset \Gamma_{V}$ and $\varphi_{V}(\tau)=\varphi(\tau)$ for all $\tau \in \Gamma$.

(b) $\Gamma_{V}$ is a partially ordered vector space and $\varphi_{V}$ is a linear order preserving map.

(c) $\left(\Gamma_{V}\right)_{V}=\Gamma_{V}$ and $\left(\varphi_{V}\right)_{V}=\varphi_{V}$.

(d) If $\Pi \subset \Gamma$, then $\Pi_{V} \subset \Gamma_{V}$.

Definition 3.6 Let $D$ be a linear subspace of $E$. $D$ is called mediated in $E$ if the following is true:

If $A$ and $B$ are countable subsets of $D$ such that $\inf A-B=0$ in $E$, then

$A$ has an infimum (and consequently $B$ has a supremum and $\inf A=\sup B$ ).

$D$ is mediated in $E$ if and only if the following requirement (equivalent with order completeness in the sense of [6], for $D=E$ ) is satisfied

If $A$ and $B$ are countable subsets of $D$ such that inf $A-B=0$ in $E$, then there exists an $h \in E$ with $B \leq h \leq A$.

We say that $E$ is mediated if $E$ is mediated in itself.

Note: if $D$ is mediated in $E$, then so is every linear subspace of $D$. Every $\sigma$-Dedekind complete $E$ is mediated, but so is $\mathbb{R}^{2}$, ordered lexicographically. Also, $c_{00}$ and $c_{0}$ are mediated in $c$, but $c$ is not mediated.

With this the following lemma is a tautology.

Lemma 3.7 Suppose $\varphi(\Gamma)$ is mediated in E. Let $f \in \Omega$. Then $f \in \Gamma_{V}$ if and only if there exist countable sets $\Lambda, \Upsilon \subset \Gamma$ with $\Lambda \leq f \leq \Upsilon$ such that

$$
\inf _{\tau \in \Upsilon, \sigma \in \Lambda} \varphi(\tau-\sigma)=0 .
$$

The next example shows that $\Gamma_{V}$ is not necessarily a Riesz space even if $E$ and $\Gamma$ are. However, see Corollary 3.10 .

Example 3.8 Consider $E=c, \Gamma=c \times c, \Omega=\ell^{\infty} \times \ell^{\infty}$. Let $\varphi: \Gamma \rightarrow c$ be given by $\varphi(f, g)=f+g$. For all $f \in \ell^{\infty}$ there are $h_{1}, h_{2}, \ldots \in c$ with $h_{n} \downarrow f$. It follows that, $\Gamma_{V}=\left\{(f, g) \in \ell^{\infty} \times \ell^{\infty}: f+g \in c\right\}$. Note that $\Gamma_{V}$ is not a Riesz space since for every $f \in \ell^{\infty}$ with $f \geq 0$ and $f \notin c$ we have $(f,-f) \in \Gamma_{V}$ but $(f,-f)^{+}=(f, 0) \notin \Gamma_{V}$.

Lemma 3.9 Suppose $\varphi(\Gamma)$ is mediated in E. Let $\Theta: \Omega \rightarrow \Omega$ be an order preserving map with the properties:

- if $\sigma, \tau \in \Gamma$ and $\sigma \leq \tau$, then $0 \leq \Theta(\tau)-\Theta(\sigma) \leq \tau-\sigma$;

- $\Theta(\Gamma) \subset \Gamma_{V}$.

Then $\Theta\left(\Gamma_{V}\right) \subset \Gamma_{V}$. 
Proof Let $f \in \Gamma_{V}$ and let $\Lambda, \Upsilon \subset \Gamma$ be countable sets with $\Lambda \leq f \leq \Upsilon$ satisfying (6). Then $\Theta(\Lambda) \leq \Theta(f) \leq \Theta(\Upsilon)$ and

$$
\inf _{\tau \in \Theta(\Upsilon), \sigma \in \Theta(\Lambda)} \varphi(\tau-\sigma)=\inf _{\tau \in \Upsilon, \sigma \in \Lambda} \varphi(\Theta(\tau)-\Theta(\sigma)) \leq \inf _{\tau \in \Upsilon, \sigma \in \Lambda} \varphi(\tau-\sigma)=0 .
$$

Corollary 3.10 Suppose that $\varphi(\Gamma)$ is mediated in E. Suppose $\Omega$ is a Riesz space and $\Gamma$ is a Riesz subspace of $\Omega$. Then so is $\Gamma_{V}$.

Proof Apply Theorem 3.9 with $\Theta(\omega)=\omega^{+}$.

3.11 If $\Gamma$ is a directed set, i.e., $\Gamma=\Gamma^{+}-\Gamma^{+}$, then so is $\Gamma_{V}$. Indeed, if $f \in \Gamma_{V}$, then there exist $\sigma, \tau \in \Gamma^{+}$such that $f \geq \tau-\sigma$ and thus $f=(f+\sigma)-\sigma \in \Gamma_{V}^{+}-\Gamma_{V}^{+}$.

3.12 In the last part of this section we will consider a situation in which $\Omega$ has some extra structure. But first we briefly consider the case where $E$ is a Banach lattice with $\sigma$-order continuous norm. As it turns out, such an $E$ is mediated (see Theorem 4.24), but is not necessarily $\sigma$-Dedekind complete (consider the Banach lattice $C(X)$ where $X$ is the one-point compactification of an uncountable discrete space). For such $E$ we describe $\Gamma_{V}$ in terms of the norm.

Theorem 3.13 Let $E$ be a Banach lattice with a $\sigma$-order continuous norm. Let $\Omega$ be a Riesz space and $\Gamma$ be a Riesz subspace of $\Omega$. For $f \in \Omega$ we have: $f \in \Gamma_{V}$ if and only iffor every $\varepsilon>0$ there exist $\sigma, \tau \in \Gamma$ with $\sigma \leq f \leq \tau$ and $\|\varphi(\tau)-\varphi(\sigma)\|<\varepsilon$.

Proof First, assume $f \in \Gamma_{V}$. As $\Gamma$ is a Riesz subspace of $\Omega$ there exist sequences $\left(\sigma_{n}\right)_{n \in \mathbb{N}}$ and $\left(\tau_{n}\right)_{n \in \mathbb{N}}$ in $\Gamma$ such that $\sigma_{n} \uparrow, \tau_{n} \downarrow$,

$$
\sigma_{n} \leq f \leq \tau_{n} \quad(n \in \mathbb{N}), \quad \sup _{n \in \mathbb{N}} \varphi\left(\sigma_{n}\right)=\inf _{n \in \mathbb{N}} \varphi\left(\tau_{n}\right)
$$

Then $\varphi\left(\tau_{n}-\sigma_{n}\right) \downarrow 0$ in $E$, so $\left\|\varphi\left(\tau_{n}\right)-\varphi\left(\sigma_{n}\right)\right\| \downarrow 0$ and we are done.

The converse: For each $n \in \mathbb{N}$, choose $\sigma_{n}, \tau_{n} \in \Gamma$ for which

$$
\sigma_{n} \leq f \leq \tau_{n}, \quad\left\|\varphi\left(\tau_{n}\right)-\varphi\left(\sigma_{n}\right)\right\| \leq n^{-1}
$$

Setting $\sigma_{n}^{\prime}=\sigma_{1} \vee \cdots \vee \sigma_{n}$ and $\tau_{n}^{\prime}=\tau_{1} \wedge \cdots \wedge \tau_{n}$ we have, for each $n \in \mathbb{N}$

$$
\sigma_{n}^{\prime}, \tau_{n}^{\prime} \in \Gamma, \quad \sigma_{n}^{\prime} \leq f \leq \tau_{n}^{\prime}
$$

If $n \geq N$, then $0 \leq \sigma_{n}^{\prime}-\sigma_{N}^{\prime} \leq f-\sigma_{N} \leq \tau_{N}-\sigma_{N}$, whence $\left\|\varphi\left(\sigma_{n}^{\prime}\right)-\varphi\left(\sigma_{N}^{\prime}\right)\right\| \leq$ $\left\|\varphi\left(\tau_{N}\right)-\varphi\left(\sigma_{N}\right)\right\| \leq N^{-1}$. Thus, the sequence $\left(\varphi\left(\sigma_{n}^{\prime}\right)\right)_{n \in \mathbb{N}}$ converges in the sense of the norm. So does $\left(\varphi\left(\tau_{n}^{\prime}\right)\right)_{n \in \mathbb{N}}$. Their limits are the same element $a$ of $E$, and, since $\sigma_{n}^{\prime} \uparrow, \tau_{m}^{\prime} \downarrow$, we see that $a=\sup _{n \in \mathbb{N}} \varphi\left(\sigma_{n}^{\prime}\right)=\inf _{m \in \mathbb{N}} \varphi\left(\tau_{m}^{\prime}\right)$. 


\subsection{In the rest of this section $\Omega$ is the collection $F^{X}$ of all maps of a set $X$ into a partially ordered vector space $F$.}

3.15 A function $g: X \rightarrow \mathbb{R}$ determines a multiplication operator $f \mapsto g f$ in $\Omega$. We investigate the collection of all functions $g$ for which

$$
f \in \Gamma_{V} \Longrightarrow g f \in \Gamma_{V}
$$

and, for given $f$, the behaviour of the map $g \mapsto \varphi_{V}(g f)$.

3.16 For an algebra of subsets of $X, \mathcal{A} \subset \mathcal{P}(X)$ we write $[\mathcal{A}]$ for the Riesz space of all $\mathcal{A}$-step functions, i.e., functions of the form $\sum_{i=1}^{n} \lambda_{i} \mathbb{1}_{A_{i}}$ for $n \in \mathbb{N}, \lambda_{i} \in \mathbb{R}$, $A_{i} \in \mathcal{A}$ for $i \in\{1, \ldots, n\}$. Define the collection of functions $[\mathcal{A}]^{o}$ by

$$
\begin{aligned}
{[\mathcal{A}]^{o}=} & \left\{f \in \mathbb{R}^{X}: \text { there are }\left(s_{n}\right)_{n \in \mathbb{N}} \text { in }[\mathcal{A}] \text { and }\left(j_{n}\right)_{n \in \mathbb{N}} \text { in }[\mathcal{A}]^{+}\right. \\
& \text {for which } \left.\left|f-s_{n}\right| \leq j_{n} \text { and } j_{n} \downarrow 0 \text { pointwise }\right\} .
\end{aligned}
$$

(This $[\mathcal{A}]^{\circ}$ is the vertical extension of $[\mathcal{A}]$ obtained by, in Definition 3.3 , choosing $E=\mathbb{R}^{X}, \Omega=\mathbb{R}^{X}, \Gamma=[\mathcal{A}], \varphi(f)=f(f \in \Gamma)$.) Note that $[\mathcal{A}]$ and $[\mathcal{A}]^{o}$ are Riesz spaces, and uniform limits of elements of $[\mathcal{A}]$ are in $[\mathcal{A}]^{o}$. (Actually, $[\mathcal{A}]^{o}$ is uniformly complete.) Furthermore, $[\mathcal{A}]^{o}$ contains every bounded function $f$ with $\{x \in X: f(x) \leq s\} \in \mathcal{A}$ for all $s \in \mathbb{R}$. In case $\mathcal{A}$ is a $\sigma$-algebra, $[\mathcal{A}]^{o}$ is precisely the collection of all bounded $\mathcal{A}$-measurable functions.

Lemma 3.17 Let $\mathcal{A} \subset \mathcal{P}(X)$ be an algebra of subsets of a set $X$. Suppose that $\left(g_{n}\right)_{n \in \mathbb{N}}$ is a sequence in $[\mathcal{A}]^{o}$ for which $g_{n} \downarrow 0$ pointwise. Then there exists a sequence $\left(j_{n}\right)_{n \in \mathbb{N}}$ in $[\mathcal{A}]$ with $j_{n} \geq g_{n}$ and $j_{n} \downarrow 0$ pointwise.

Proof For all $n \in \mathbb{N}$ there exists a sequence $\left(s_{n k}\right)_{k \in \mathbb{N}}$ in $[\mathcal{A}]$ with $s_{n k} \geq g_{n}$ for all $k \in \mathbb{N}$ and $s_{n k} \downarrow_{k} g_{n}$ pointwise. Since $\left(g_{n}\right)_{n \in \mathbb{N}}$ is a decreasing sequence, we have $s_{m k} \geq g_{n}$ for all $m \leq n$ and all $k \in \mathbb{N}$. Hence $j_{n}:=\inf _{m, k \leq n} s_{m k}$ is an element in $[\mathcal{A}]$ with $j_{n} \geq g_{n}$. Clearly $j_{n} \downarrow$ and $\inf _{n \in \mathbb{N}} j_{n}=\inf _{n \in \mathbb{N}} \inf _{m, k \leq n} s_{m k}=\inf _{n \in \mathbb{N}} \inf _{k \in \mathbb{N}} s_{n k}=$ $\inf _{n \in \mathbb{N}} g_{n}=0$.

The following lemma is a consequence of Lemma 3.9.

Lemma 3.18 Define the algebra

$$
\mathcal{A}=\left\{A \subset X: f \mathbb{1}_{A} \in \Gamma \text { for } f \in \Gamma\right\} .
$$

If $\varphi(\Gamma)$ is mediated in $E$, then

$$
f \mathbb{1}_{A} \in \Gamma_{V} \quad\left(f \in \Gamma_{V}, A \in \mathcal{A}\right) .
$$

Definition 3.19 $E$ is called Archimedean ${ }^{3}$ (see Peressini [8]) if for all $a, b \in E$ the following holds: if $n a \leq b$ for all $n \in \mathbb{N}$, then $a \leq 0$.

\footnotetext{
3 In some places, e.g., Birkhoff [7], the term 'integrally closed' is used.
} 
Definition 3.20 A sequence $\left(a_{n}\right)_{n \in \mathbb{N}}$ in $E$ is called order convergent to an element $a \in E$ if there exists a sequence $\left(h_{n}\right)_{n \in \mathbb{N}}$ in $E^{+}$with $h_{n} \downarrow 0$ and $-h_{n} \leq a-a_{n} \leq h_{n}$. Notation: $a_{n} \stackrel{o}{\rightarrow} a$.

Theorem 3.21 Let $\mathcal{A}$ be as in (13). Suppose that $E$ is Archimedean, $\Gamma$ is directed and $\varphi(\Gamma)$ is mediated in E. Furthermore assume $\varphi$ has the following continuity property.

$$
\begin{aligned}
& \text { If } A_{1}, A_{2}, \ldots \text { in } \mathcal{A} \text { are such that } A_{1} \supset A_{2} \supset \cdots \text { and } \bigcap_{n \in \mathbb{N}} A_{n}=\emptyset \text {, } \\
& \text { then } \varphi\left(f \mathbb{1}_{A_{n}}\right) \downarrow 0 \text { for all } f \in \Gamma^{+} .
\end{aligned}
$$

(a) $g f \in \Gamma_{V}$ for all $g \in[\mathcal{A}]^{o}$ and all $f \in \Gamma_{V}$.

(b) Let $g \in[\mathcal{A}]^{o}$ and let $\left(g_{n}\right)_{n \in \mathbb{N}}$ be a sequence in $[\mathcal{A}]^{\circ}$ for which there is a sequence $\left(j_{n}\right)_{n \in \mathbb{N}}$ in $[\mathcal{A}]^{o+}$ with $-j_{n} \leq g_{n}-g \leq j_{n}$ and $j_{n} \downarrow 0$ pointwise. Then

$$
\varphi_{V}\left(g_{n} f\right) \stackrel{o}{\rightarrow} \varphi_{V}(g f) \quad\left(f \in \Gamma_{V}\right) .
$$

(Order convergence in the sense of $E$.)

Proof We first prove the following:

$(\star)$ Let $f \in \Gamma_{V}^{+}$. Let $\left(g_{n}\right)_{n \in \mathbb{N}}$ be a sequence in $[\mathcal{A}]^{o}$ for which $g_{n} f \in \Gamma_{V}$ for all $n \in \mathbb{N}$ and $g_{n} \downarrow 0$ pointwise. Then

$$
\varphi_{V}\left(g_{n} f\right) \downarrow 0 .
$$

Let $\sigma \in \Gamma^{+}, \sigma \geq f$. It follows from Lemma 3.17 that we may assume $g_{n} \in[\mathcal{A}]$ for all $n \in \mathbb{N}$. For all $n \in \mathbb{N}$ we have $0 \leq \varphi_{V}\left(g_{n} f\right) \leq \varphi_{V}\left(g_{n} \sigma\right)$, so we are done if $\varphi_{V}\left(g_{n} \sigma\right) \downarrow 0$.

Let $h \in E, h \leq \varphi_{V}\left(g_{n} \sigma\right)$ for all $n \in \mathbb{N}$; we prove $h \leq 0$.

Take $\varepsilon>0$. For each $n \in \mathbb{N}$, set $A_{n}=\left\{x \in X: g_{n}(x) \geq \varepsilon\right\}$. Then $A_{n} \in \mathcal{A}$ for $n \in \mathbb{N}$ and $A_{1} \supset A_{2} \supset \cdots$ and $\bigcap_{n \in \mathbb{N}} A_{n}=\emptyset$. Putting $M=\left\|g_{1}\right\|_{\infty}$ we see that

$$
g_{n} \leq \varepsilon \mathbb{1}_{X}+M \mathbb{1}_{A_{n}} \quad(n \in \mathbb{N}),
$$

whence

$$
h \leq \varphi_{V}\left(g_{n} \sigma\right) \leq \varepsilon \varphi(\sigma)+M \varphi\left(\mathbb{1}_{A_{n}} \sigma\right) \quad(n \in \mathbb{N}) .
$$

By the continuity property of $\varphi, h \leq \varepsilon \varphi(\sigma)$. As this is true for each $\varepsilon>0$ and $E$ is Archimedean, we obtain $h \leq 0$.

(a) Since $\Gamma_{V}$ is directed (see 3.11) it is sufficient to consider $f \in \Gamma_{V}^{+}$. Let $g \in[\mathcal{A}]^{o}$. There are sequences of step functions $\left(h_{n}\right)_{n \in \mathbb{N}}$ and $\left(j_{n}\right)_{n \in \mathbb{N}}$ for which $h_{n} \uparrow g, j_{n} \downarrow g$ and thus $j_{n}-h_{n} \downarrow 0$. By Lemma $3.18 h_{n} f, j_{n} f \in \Gamma_{V}$ for all $n \in \mathbb{N}$. Then $h_{n} f \leq$ $g f \leq j_{n} f$ for $n \in \mathbb{N}$ and $\inf _{n \in \mathbb{N}} \varphi_{V}\left(\left(j_{n}-h_{n}\right) f\right)=0$ by $(\star)$. By Lemmas 3.7 and 3.5(c) we obtain that $g f \in \Gamma_{V}$. 
(b) It is sufficient to consider $f \in \Gamma_{V}^{+}$. By (a) we may also assume $g=0$. But then (b) follows from $(\star)$.

Remark 3.22 Consider the situation in Theorem 3.21. Suppose $\mathcal{B} \subset \mathcal{A}$ is a $\sigma$-algebra. Then all bounded $\mathcal{B}$-measurable functions lie in $[\mathcal{A}]^{o}$. If $\left(g_{n}\right)_{n \in \mathbb{N}}$ is a bounded sequence of bounded $\mathcal{B}$-measurable functions that converges pointwise to a function $g$, then the condition of Theorem 3.21(b) is satisfied.

Remark 3.23 In the next section we will consider a situation similar to the one of Theorem 3.21, in which $\mathcal{A}$ is replaced by a subset $\mathcal{I}$ that is closed under taking finite intersections. We will also adapt the continuity property on $\varphi$ (see 4.3).

\section{The lateral extension}

The construction described in Definition 3.3 is reminiscent of the Riemann integral and, indeed, the Riemann integral is a special case (see Example 3.4).

In the present section we consider a type of extension, analogous to the improper Riemann integral. One usually defines the improper integral of a function $f$ on $[0, \infty)$ to be

$$
\lim _{s \rightarrow \infty} \int_{0}^{s} f(x) \mathrm{d} x,
$$

approximating the domain, not the values of $f$.

For our purposes a more convenient description of the same integral would be

$$
\sum_{n=1}^{\infty} \int_{a_{n}}^{a_{n+1}} f(x) \mathrm{d} x,
$$

where $0=a_{1}<a_{2}<\cdots$ and $a_{n} \rightarrow \infty$. Here the domain is split up into manageable pieces.

Splitting up the domain is the basic idea we develop in this section. (This may explain our use of the terms "vertical" and "lateral".)

Throughout this section, $X$ is a set, $E$ and $F$ are partially ordered vector spaces, $\Gamma$ is a directed ${ }^{4}$ linear subspace of $\mathbf{F}^{\mathbf{X}}$, and $\varphi$ is a linear order preserving map $\Gamma \rightarrow E$. (With $\Omega=F^{X}$, all considerations of Sect. 3 are applicable.)

$$
\begin{aligned}
& \Gamma \subset F^{X} \\
& \qquad \underset{E}{\downarrow}
\end{aligned}
$$

Furthermore, $\mathcal{I}$ is a collection of subsets of $X$, closed under taking finite intersections. See Definitions 4.1 and 4.2 for two more assumptions.

\footnotetext{
${ }^{4}$ For the construction of the lateral extension, one does not need to assume that $\Gamma$ is directed. However, as one can see later on in the construction, the only part of $\Gamma$ that matters for the extension is $\Gamma^{+}-\Gamma^{+}$.
} 
As a shorthand notation, if $\left(a_{n}\right)_{n \in \mathbb{N}}$ is a sequence in $E^{+}$and $\left\{\sum_{n=1}^{N} a_{n}: N \in \mathbb{N}\right\}$ has a supremum, we denote this supremum by

$$
\sum_{n} a_{n}
$$

Definition 4.1 A disjoint sequence $\left(A_{n}\right)_{n \in \mathbb{N}}$ of elements in $\mathcal{I}$ whose union is $X$ is called a partition If $\left(A_{n}\right)_{n \in \mathbb{N}}$ and $\left(B_{n}\right)_{n \in \mathbb{N}}$ are partitions and for all $n \in \mathbb{N}$ there exists an $m \in \mathbb{N}$ for which $B_{n} \subset A_{m}$, then $\left(B_{n}\right)_{n \in \mathbb{N}}$ is called a refinement of $\left(A_{n}\right)_{n \in \mathbb{N}}$. Note that if $\left(A_{n}\right)_{n \in \mathbb{N}}$ and $\left(B_{n}\right)_{n \in \mathbb{N}}$ are partitions then there exists a refinement of both $\left(A_{n}\right)_{n \in \mathbb{N}}$ and $\left(B_{n}\right)_{n \in \mathbb{N}}$ (e.g., a partition that consists of all sets of the form $A_{n} \cap B_{m}$ with $n, m \in \mathbb{N}$ ). We assume that there exists at least one partition.

Definition 4.2 We call a linear subspace $\Delta$ of $F^{X}$ stable (under $\mathcal{I}$ ) if

$$
f \mathbb{1}_{A} \in \Delta \quad(f \in \Delta, A \in \mathcal{I}) .
$$

If $\Delta$ is a stable space, then a linear and order preserving map $\omega: \Delta \rightarrow E$ is said to be laterally extendable if for all partitions $\left(A_{n}\right)_{n \in \mathbb{N}}$

$$
\omega(f)=\sum_{n} \omega\left(f \mathbb{1}_{A_{n}}\right) \quad(\operatorname{see}(22)) \quad\left(f \in \Delta^{+}\right) .
$$

\section{We assume $\Gamma$ is stable and $\varphi$ is laterally extendable.}

4.3 In the situation of Theorem 3.21 we can choose $\mathcal{I}=\mathcal{A}$; then (15) is precisely the lateral extendability of $\varphi$.

Example 4.4 For any partially ordered vector space $F$ and a linear subspace $E \subset F$, the following choices lead to a system fulfilling all of our assumptions: $X=\mathbb{N}$, $\mathcal{I}=\mathcal{P}(\mathbb{N}), \Gamma=c_{00}[E]\left(\right.$ see Sect. 2), $\varphi(f)=\sum_{n \in \mathbb{N}} f(n)$ for $f \in \Gamma$.

Definition 4.5 Let $\Delta$ be a stable subspace of $F^{X}$ and let $\omega: \Delta \rightarrow E$ be a laterally extendable linear order preserving map. Let $\left(A_{n}\right)_{n \in \mathbb{N}}$ be a partition, and $f: X \rightarrow F$. We call $\left(A_{n}\right)_{n \in \mathbb{N}}$ a partition for $f$ (occasionally $\Delta$-partition for $f$ ) if

$$
f \mathbb{1}_{A_{n}} \in \Delta \quad(n \in \mathbb{N}) .
$$

A function $f: X \rightarrow F$ is said to be a partially in $\Delta$ if there exists a partition for $f$. For $f: X \rightarrow F^{+},\left(A_{n}\right)_{n \in \mathbb{N}}$ is called a $\omega$-partition for $f$ if it is a partition for $f$ and if

$$
\sum_{n} \omega\left(f \mathbb{1}_{A_{n}}\right) \text { exists. }
$$

A function $f: X \rightarrow F^{+}$that is partially in $\Delta$ is called laterally $\omega$-integrable if there exists a $\omega$-partition for $f$. 
Example 4.6 Consider the situation of Example 4.4. A function $x: \mathbb{N} \rightarrow F$ is partially in $\Gamma$ if and only if $x_{n} \in E$ for every $n \in \mathbb{N}$. If $x \geq 0$, then $x$ is laterally integrable if $x_{n} \in E$ for every $n \in \mathbb{N}$ and $\sum_{n} x_{n}$ exists in $E$.

4.7 Naturally, we wish to use (26) to define an integral for $f$. For that we have to show the supremum to be independent of the choice of the partition $\left(A_{n}\right)_{n \in \mathbb{N}}$.

Lemma 4.8 (a) Let $f: X \rightarrow F$ and let $\left(A_{n}\right)_{n \in \mathbb{N}}$ be a partition for $f$. If $\left(B_{n}\right)_{n \in \mathbb{N}}$ is a partition that is a refinement of $\left(A_{n}\right)_{n \in \mathbb{N}}$, then $\left(B_{n}\right)_{n \in \mathbb{N}}$ is a partition for $f$.

(b) Let $f: X \rightarrow F^{+}$and let $\left(A_{n}\right)_{n \in \mathbb{N}}$ and $\left(B_{m}\right)_{m \in \mathbb{N}}$ be partitions for $f$. Then the sets

$$
\left\{\sum_{n=1}^{N} \varphi\left(f \mathbb{1}_{A_{n}}\right): N \in \mathbb{N}\right\} \text { and }\left\{\sum_{m=1}^{M} \varphi\left(f \mathbb{1}_{B_{m}}\right): M \in \mathbb{N}\right\}
$$

have the same upper bounds in $E$.

Proof We leave the proof of (a) to the reader. Let $u$ be an upper bound for the set $\left\{\sum_{n=1}^{N} \varphi\left(f \mathbb{1}_{A_{n}}\right): N \in \mathbb{N}\right\}$; it suffices to prove that $u$ is an upper bound for $\left\{\sum_{m=1}^{M} \varphi\left(f \mathbb{1}_{B_{m}}\right): M \in \mathbb{N}\right\}$. Take $M \in \mathbb{N}$; we are done if $u \geq \sum_{m=1}^{M} \varphi\left(f \mathbb{1}_{B_{m}}\right)$, i.e., if $u \geq \varphi\left(f \mathbb{1}_{B}\right)$ where $B=B_{1} \cup \cdots \cup B_{M}$. But $f \mathbb{1}_{B} \in \Gamma$ so $\varphi\left(f \mathbb{1}_{B}\right)=$ $\sum_{n} \varphi\left(f \mathbb{1}_{B} \mathbb{1}_{A_{n}}\right)=\sup _{N \in \mathbb{N}} \sum_{n=1}^{N} \varphi\left(f \mathbb{1}_{B} \mathbb{1}_{A_{n}}\right)$, whereas, for each $N \in \mathbb{N}$

$$
\sum_{n=1}^{N} \varphi\left(f \mathbb{1}_{B} \mathbb{1}_{A_{n}}\right) \leq \sum_{n=1}^{N} \varphi\left(f \mathbb{1}_{A_{n}}\right) \leq u
$$

Theorem 4.9 Let $f: X \rightarrow F^{+}$be laterally $\varphi$-integrable. Then every partition for $f$ is a $\varphi$-partition for $f$. There exists an a $\in E^{+}$such that for every partition $\left(A_{n}\right)_{n \in \mathbb{N}}$ for $f$,

$$
a=\sum_{n} \varphi\left(f \mathbb{1}_{A_{n}}\right)
$$

If $f \in \Gamma^{+}$, then $a=\varphi(f)$.

Proof This is a consequence of Lemma 4.8(b).

Definition 4.10 For a laterally $\varphi$-integrable $f: X \rightarrow F^{+}$we call the element $a \in E^{+}$ for which (29) holds its $\varphi_{L}$-integral and denote it by $\varphi_{L}(f)$. For the moment, denote by $\left(\Gamma^{+}\right)_{L}$ the set of all laterally $\varphi$-integrable functions $f: X \rightarrow F^{+}$. We proceed to extend $\varphi_{L}$ to a linear function defined on the linear hull of $\left(\Gamma^{+}\right)_{L}$, see Definition 4.14.

4.11 The assumptions that $\Gamma$ is stable and $\varphi$ is laterally extendable are crucial for the fact that the $\varphi_{L}$-integral of a laterally $\varphi$-integrable function is independent of the choice of a $\varphi$-partition (see Lemma 4.8(b)). 
4.12 We will use the following rules for a partially ordered vector space $E$ :

$$
\begin{aligned}
a_{n} \uparrow a, b_{n} \uparrow b & \Longrightarrow a_{n}+b_{n} \uparrow a+b & & \left(a_{n}, b_{n}, a, b \in E\right), \\
a_{n} \uparrow, b_{n} \uparrow b, a_{n}+b_{n} \uparrow a+b & \Longrightarrow a_{n} \uparrow a & & \left(a_{n}, b_{n}, a, b \in E\right) .
\end{aligned}
$$

4.13 (Extending $\left.\varphi_{L}\right)$ Define $\Gamma_{L}=\left\{f_{1}-f_{2}: f_{1}, f_{2} \in\left(\Gamma^{+}\right)_{L}\right\}$.

Step 1 Let $f, g \in\left(\Gamma^{+}\right)_{L}$. There exists an $\left(A_{n}\right)_{n \in \mathbb{N}}$ that is a $\varphi$-partition for $f$ and for $g$. By defining $a_{N}=\sum_{n=1}^{N} \varphi\left(f \mathbb{1}_{A_{n}}\right)$ and $b_{N}=\sum_{n=1}^{N} \varphi\left(g \mathbb{1}_{A_{n}}\right)$ for $N \in \mathbb{N}$, by (30) we obtain $f+g \in\left(\Gamma^{+}\right)_{L}$ with $\varphi_{L}(f+g)=\varphi_{L}(f)+\varphi_{L}(g)$.

Consequently, $\Gamma_{L}$ is a vector space, containing $\left(\Gamma^{+}\right)_{L}$.

Step 2 If $g_{1}, g_{2}, h_{1}, h_{2} \in\left(\Gamma^{+}\right)_{L}$ and $g_{1}-g_{2}=h_{1}-h_{2}$, then $g_{1}+h_{2}=g_{2}+h_{1}$ so that, by the above, $\varphi_{L}\left(g_{1}\right)-\varphi_{L}\left(h_{1}\right)=\varphi_{L}\left(g_{2}\right)-\varphi_{L}\left(h_{2}\right)$.

Hence, $\varphi_{L}$ extends to a linear function $\Gamma_{L} \rightarrow E$ (also denoted by $\varphi_{L}$ ).

Step 3 Let $f, g \in\left(\Gamma^{+}\right)_{L}$ and $f \leq g$. By defining $a_{N}$ and $b_{N}$ as in step 1 and $c_{N}=b_{N}-a_{N}$, by (31) we infer that $g-f \in\left(\Gamma^{+}\right)_{L}$.

Thus, if $f \in \Gamma_{L}$ and $f \geq 0$, then $f \in\left(\Gamma^{+}\right)_{L}$. Briefly: $\left(\Gamma^{+}\right)_{L}$ is $\Gamma_{L}^{+}$, the positive part of $\Gamma_{L}$.

Definition 4.14 A function $f: X \rightarrow F$ is called laterally $\varphi$-integrable if $f \in \Gamma_{L}$ (see 4.13), i.e., if there exist $f_{1}, f_{2} \in\left(\Gamma^{+}\right)_{L}$ for which $f=f_{1}-f_{2}$. The $\varphi_{L}$-integral of such a function is defined by $\varphi_{L}(f)=\varphi_{L}\left(f_{1}\right)-\varphi_{L}\left(f_{2}\right)$.

$\varphi_{L}$ is a function $\Gamma_{L} \rightarrow E$ and is called the lateral extension of $\varphi$. The set of laterally $\varphi$-integrable functions, $\Gamma_{L}$, is called the lateral extension of $\Gamma$ under $\varphi$.

Note that, thanks to Step 3 of 4.13, this definition of "laterally $\varphi$-integrable" does not conflict with the one given in Definition 4.10.

4.15 Like for the vertical extension, we have the following elementary observations:

(a) $\Gamma \subset \Gamma_{L}^{5}$ and $\varphi_{L}(\tau)=\varphi(\tau)$ for all $\tau \in \Gamma$.

(b) $\Gamma_{L}$ is a directed partially ordered vector space and $\varphi_{L}$ is a linear order preserving function on $\Gamma_{L}$.

(c) If $\Pi$ is a directed linear subspace of $F^{X}$ and $\Pi \subset \Gamma$, then $\Pi_{L} \subset \Gamma_{L}$.

$\left(\left(\Gamma_{L}\right)_{L}\right.$ is not so easy. See Theorem 4.18 and Example 4.19.)

In case $E$ is a Banach lattice with $\sigma$-order continuous norm, for $\Gamma_{L}^{+}$we have an analogue of Theorem 3.13.

Lemma 4.16 Suppose $E$ is a Banach lattice with $\sigma$-order continuous norm. Let $f$ : $X \rightarrow F^{+}$. Then $f$ lies in $\Gamma_{L}^{+}$if and only if there exists a $\Gamma$-partition $\left(A_{n}\right)_{n \in \mathbb{N}}$ for $f$ such that the sequence $\left(\varphi\left(f \mathbb{1}_{A_{n}}\right)\right)_{n \in \mathbb{N}}$ has a sum in the sense of the norm, in which case $\varphi_{L}(f)$ is this sum.

Proof The "only if" part follows by definition of $\Gamma_{L}$ and the $\sigma$-order continuity of the norm. For the "if" part; this follows from the fact that if $a_{n} \uparrow$ and $\left\|a_{n}-a\right\| \rightarrow 0$ for $a, a_{1}, a_{2}, \ldots \in E$, then $a_{n} \uparrow a$.

\footnotetext{
${ }^{5}$ Note that for this inclusion it is necessary that $\Gamma$ be directed.
} 
We will now investigate conditions under which $\varphi_{L}$ and $\varphi_{V}$ themselves are laterally extendable. (For that, their domains have to be able to play the role of $\Gamma$, so they have to be stable.) First a useful lemma:

Lemma 4.17 Let $f \in \Gamma_{L}$. Then there exists a partition $\left(A_{n}\right)_{n \in \mathbb{N}}$ for $f$ such that every refinement $\left(B_{m}\right)_{m \in \mathbb{N}}$ of it (is a partition for $f$ and) has this property:

$$
h \in E, h \geq \sum_{m=1}^{M} \varphi\left(f \mathbb{1}_{B_{m}}\right) \text { for all } M \in \mathbb{N} \Longrightarrow h \geq \varphi_{L}(f) .
$$

Proof Write $f=f_{1}-f_{2}$ with $f_{1}, f_{2} \in \Gamma_{L}^{+}$. Let $\left(A_{n}\right)_{n \in \mathbb{N}}$ be a partition for $f_{1}$ and $f_{2}$, and let $\left(B_{m}\right)_{m \in \mathbb{N}}$ be a refinement of $\left(A_{n}\right)_{n \in \mathbb{N}}$. Note that $\left(B_{m}\right)_{m \in \mathbb{N}}$ is a partition for $f_{1}$ and $f_{2}$. Let $h$ be an upper bound for $\left\{\sum_{m=1}^{M} \varphi\left(f \mathbb{1}_{B_{m}}\right): M \in \mathbb{N}\right\}$ in $E$. For all $M \in \mathbb{N}$,

$$
h+\sum_{m=1}^{M} \varphi\left(f_{2} \mathbb{1}_{B_{m}}\right) \geq \sum_{m=1}^{M} \varphi\left(f \mathbb{1}_{B_{m}}\right)+\sum_{m=1}^{M} \varphi\left(f_{2} \mathbb{1}_{B_{m}}\right)=\sum_{m=1}^{M} \varphi\left(f_{1} \mathbb{1}_{B_{m}}\right) .
$$

Taking the supremum over $M$ yields $h+\varphi_{L}\left(f_{2}\right) \geq \varphi_{L}\left(f_{1}\right)$, i.e., $h \geq \varphi_{L}(f)$.

Theorem 4.18 (a) Suppose $\Gamma_{L}$ is stable. Then $\varphi_{L}$ is laterally extendable, i.e.,

$$
\varphi_{L}(f)=\sum_{n} \varphi_{L}\left(f \mathbb{1}_{A_{n}}\right)
$$

for every $f \in \Gamma_{L}^{+}$and every $\varphi_{L}$-partition $\left(A_{n}\right)_{n \in \mathbb{N}}$ for $f$. Therefore $\left(\Gamma_{L}\right)_{L}=\Gamma_{L}$ and $\left(\varphi_{L}\right)_{L}=\varphi_{L}$.

(b) Suppose $\Gamma_{V}$ is stable. Then $\varphi_{V}$ is laterally extendable. $\left(\right.$ For $\left(\Gamma_{V}\right)_{L}$ see Sect.5.)

Proof (a) Let $f \in \Gamma_{L}^{+}$and let $\left(B_{n}\right)_{n \in \mathbb{N}}$ be a $\varphi_{L}$-partition for $f$. Let $\left(A_{n}\right)_{n \in \mathbb{N}}$ be the partition for $f$ as in Lemma 4.17. Then form a common refinement of $\left(B_{n}\right)_{n \in \mathbb{N}}$ and $\left(A_{n}\right)_{n \in \mathbb{N}}$ and apply Lemma 4.17.

(b) Let $f \in \Gamma_{V}^{+}$and let $\left(A_{n}\right)_{n \in \mathbb{N}}$ be a partition. Let $h \in E, h \geq \sum_{n=1}^{N} \varphi_{V}\left(f \mathbb{1}_{A_{n}}\right)$ for every $N \in \mathbb{N}$. We wish to prove $h \geq \varphi_{V}(f)$, which will be the case if $h \geq \varphi(\sigma)$ for every $\sigma \in \Gamma$ with $\sigma \leq f$. For that apply Lemma 4.17 to $\sigma$.

The following shows that $\Gamma_{L}$ may not be stable, in which case there is no $\left(\Gamma_{L}\right)_{L}$. (However, see Theorem 4.25(a).)

Example 4.19 Consider the situation in Example 4.4 and assume there is an $a: \mathbb{N} \rightarrow$ $E^{+}$such that $\sum_{n} a_{n}$ exists in $F$ and $\sum_{n} a_{2 n}$ does not (e.g. $E=F=c$ and $a_{n}=$ $\left.e_{n}=\mathbb{1}_{\{n\}}\right)$. By Example $4.6 a$ lies in $\Gamma_{L}$ but $b=\left(0, a_{2}, 0, a_{4}, \ldots\right)$ does not; but $b=a \mathbb{1}_{\{2,4,6, \ldots\}}$ and $\{2,4,6, \ldots\} \in \mathcal{I}$. (Actually, the existence of such an $a: \mathbb{N} \rightarrow E^{+}$ is equivalent to $E$ not being "splitting" in $F$; see Definition 4.21 and (36).)

Remark 4.20 $\Gamma_{V}$ may not be stable either. With $E=c, F=\ell^{\infty}, X=\{1,2\}$, $\Gamma=c \times c$ and $\varphi(f, g)=f+g$ (as in Example 3.8), the space $\Gamma_{V}$ is not stable for $\mathcal{I}=\mathcal{P}(X)$. 
Definition 4.21 Let $D$ be a linear subspace of $E$. $D$ is called splitting in $E$ if the following is true:

$$
\begin{aligned}
& \text { If }\left(a_{n}\right)_{n \in \mathbb{N}} \text { and }\left(b_{n}\right)_{n \in \mathbb{N}} \text { are sequences in } D \text { with } 0 \leq a_{n} \leq b_{n} \text { for } n \in \mathbb{N} \\
& \text { and } \sum_{n} b_{n} \text { exists in } E \text {, then so does } \sum_{n} a_{n} .
\end{aligned}
$$

It is not difficult to see that $D$ is splitting in $E$ if and only if

$$
\begin{aligned}
& \text { If }\left(a_{n}\right)_{n \in \mathbb{N}} \text { is a sequence in } D^{+} \text {and } \sum_{n} a_{n} \text { exists in } E, \\
& \text { then so does } \sum_{n} \mathbb{1}_{A}(n) a_{n} \text { for all } A \subset \mathbb{N} \text {. }
\end{aligned}
$$

If $D$ is splitting in $E$, then so is every linear subspace of $D$. If $E$ is $\sigma$-Dedekind complete, then $E$ is also splitting. More generally, $D$ is splitting in $E$ if every bounded increasing sequence in $D$ has a supremum in $E$. Also, $\mathbb{R}^{2}$ with the lexicographical ordering is splitting.

In Theorem 4.25 we will see what is the use of this concept. First, we have a look at the connection between "splitting" and "mediated".

Lemma 4.22 Suppose D is a linear subspace of E. Consider the condition:

$$
\begin{aligned}
& \text { For all sequences }\left(a_{n}\right)_{n \in \mathbb{N}},\left(b_{n}\right)_{n \in \mathbb{N}} \operatorname{in} D: \\
& a_{n} \downarrow, b_{n} \uparrow, \inf _{n \in \mathbb{N}} a_{n}-b_{n}=0 \Longrightarrow \inf _{n \in \mathbb{N}} a_{n}=\sup _{n \in \mathbb{N}} b_{n} .
\end{aligned}
$$

(The infima and suprema in (37) are to be taken in E.) If D is either splitting or mediated in E, then (37) holds. Conversely, (37) implies that $D$ is splitting if $D=E$, whereas (37) implies that $D$ is mediated in $E$ if $E$ is a Riesz space and $D$ is a Riesz subspace of $E$.

Proof It will be clear that mediatedness implies (37) and vice versa if $E$ is a Riesz space and $D$ a Riesz subspace of $E$.

If $D$ is splitting in $E$ and $a_{n} \downarrow, b_{n} \uparrow$ and inf $a_{n}-b_{n}=0$, then $\sum_{n} b_{n+1}-b_{n}+$ $a_{n}-a_{n+1}=a_{1}-b_{1}$. Hence (37) holds.

Suppose $D=E$ and (37) holds. Let $\left(a_{n}\right)_{n \in \mathbb{N}}$ and $\left(b_{n}\right)_{n \in \mathbb{N}}$ be sequences in $D$ with $0 \leq a_{n} \leq b_{n}$ for $n \in \mathbb{N}$ such that $\sum_{n} b_{n}$ exists. Let $z=\sum_{n} b_{n}, A_{n}=\sum_{i=1}^{n} a_{i}$, $C_{n}=\sum_{i=1}^{n} b_{i}-a_{i}$ for $n \in \mathbb{N}$. Then $A_{n} \uparrow, C_{n} \uparrow$ and $z-C_{n}-A_{n} \downarrow 0$ (note that $\left.z-C_{n} \in D\right)$. Hence $\sup _{n \in \mathbb{N}} A_{n}=\sum_{n} a_{n}$ exists.

4.23 (a) If $E$ is a Riesz space, then every splitting Riesz subspace is mediated in $E$.

(b) If $E$ is mediated, then it is splitting. The converse is also true if $E$ is a Riesz space.

(c) $c_{00}$ is mediated in $c$, not splitting in $c$ (with $D=E=c$ also (37) is not satisfied).

(d) If $D$ is the space of all polynomial functions on $[0,1]$ with degree at most 2 and $E=C[0,1]$, then $D$ is splitting in $E$, but not mediated in $E$. (Actually, $D$ is splitting, but not mediated.) 
$D$ is splitting (and satisfies (37) with $E=D$ ): if $u_{n} \in E^{+}, u_{n} \uparrow$ and $u_{n} \leq \mathbb{1}$, then $\left|u_{n}(x)-u_{n}(y)\right| \leq 4|x-y|$ as can be concluded from the postscript in Example 5.15. Therefore the pointwise supremum is continuous. It is even in $D$ since $u_{n}(x)=a_{n} x^{2}+b_{n} x+c_{n}$, where $a_{n}, b_{n}, c_{n}$ are linear combinations of $u_{n}(0), u_{n}\left(\frac{1}{2}\right), u_{n}(1)$ (see also the postscript in Example 5.15).

$D$ is not mediated: for example one can find countable $A, B \subset E$ for which $\mathbb{1}_{\left[\frac{1}{2}, 1\right]}$ is pointwise the infimum of $A$ and $\mathbb{1}_{\left(\frac{1}{2}, 1\right]}$ is pointwise the supremum of $B$, then inf $A-B=0$, but there is no $h \in E$ with $B \leq h \leq A$.

Theorem 4.24 Let $E$ be a Banach lattice with $\sigma$-order continuous norm. Then $E$ is both mediated and splitting.

Proof Suppose $a_{n}, b_{n} \in E$ with $0 \leq a_{n} \leq b_{n}$ for $n \in \mathbb{N}$. Suppose that $\left\{\sum_{n=1}^{N} b_{n}\right.$ : $N \in \mathbb{N}\}$ has a supremum $s$ in $E$. We prove that $\left\{\sum_{n=1}^{N} a_{n}: N \in \mathbb{N}\right\}$ has a supremum in $E$. Since the norm is $\sigma$-order continuous, we have $\left\|s-\sum_{n=1}^{N} b_{n}\right\| \rightarrow 0$. In particular we get that for all $\varepsilon>0$ there exists an $N \in \mathbb{N}$ such that for all $n, m \geq N$ with $m>n$ we have $\left\|\sum_{i=n}^{m} b_{i}\right\|<\varepsilon$ and thus $\left\|\sum_{i=n}^{m} a_{i}\right\|<\varepsilon$. From this we infer that $\left(\sum_{n=1}^{N} a_{n}\right)_{N \in \mathbb{N}}$ converges in norm. Therefore it has a supremum in $E$. Thus $E$ is splitting. By Lemma 4.22 $E$ is mediated.

Theorem 4.25 (a) $\varphi(\Gamma)$ splitting in $E \Longrightarrow \Gamma_{L}$ is stable and $\varphi_{L}$ is laterally extendable.

(b) $\varphi(\Gamma)$ mediated in $E \Longrightarrow \Gamma_{V}$ is stable and $\varphi_{V}$ is laterally extendable.

(c) $\varphi(\Gamma)$ splitting in $E$ and $\varphi_{L}\left(\Gamma_{L}\right)$ mediated in $E \Longrightarrow\left(\Gamma_{L}\right)_{V}$ is stable and $\left(\varphi_{L}\right)_{V}$ is laterally extendable.

Proof (a) Let $f \in \Gamma_{L}, B \in \mathcal{I}$; we prove $f \mathbb{1}_{B} \in \Gamma_{L}$. (This is sufficient by Theorem 4.18(a).) Without loss of generality, assume $f \geq 0$. Choose a $\varphi$-partition $\left(A_{n}\right)_{n \in \mathbb{N}}$ for $f$. Now apply (35) to

$$
a_{n}:=\varphi\left(f \mathbb{1}_{A_{n} \cap B}\right), \quad b_{n}:=\varphi\left(f \mathbb{1}_{A_{n}}\right) \quad(n \in \mathbb{N}) .
$$

(b) follows from Lemma 3.18 and Theorem 4.18(b).

(c) By (a) $\Gamma_{L}$ is stable and $\varphi_{L}$ is laterally extendable. Hence we can apply (b) to $\Gamma_{L}$ and $\varphi_{L}$ (instead of $\Gamma$ and $\varphi$ ) and obtain (c).

4.26 To some extent, the assumption of Theorem 4.25(a) is minimal.

Indeed, in the situation of Example 4.4, we see that $\Gamma_{L}$ is stable if and only if $E$ (which is $\varphi(\Gamma)$ ) is splitting in $F$ (see (36)).

In Theorem 4.25(c) we assumed that $\varphi_{L}\left(\Gamma_{L}\right)$ (and thus also $\varphi(\Gamma)$ ) was mediated in $E$. It may happen that $\varphi(\Gamma)$ is mediated in $E$, but $\varphi_{L}\left(\Gamma_{L}\right)$ is not, as Example 4.27 illustrates. However, splitting is preserved under the lateral extension and mediation is preserved under the vertical extension, see Theorem 4.28.

Example 4.27 Let $X=\mathbb{N}, \mathcal{I}=\mathcal{P}(\mathbb{N}), E=F=c$. Let $\Gamma=c_{00}\left[c_{00}\right]$ (see Sect. 2) and $\varphi: \Gamma \rightarrow E$ be given by $\varphi(f)=\sum_{n \in \mathbb{N}} f(n)$. Then $\varphi(\Gamma)=c_{00}$, which is mediated in 
c. A function $f: \mathbb{N} \rightarrow c$ is partially in $\Gamma$ if and only if $f(\mathbb{N}) \subset c_{00}$. For $x \in c^{+}$the function given by $f(n)=x(n) \mathbb{1}_{\{n\}}$ for $n \in \mathbb{N}$ lies in $\Gamma_{L}$, and $\varphi_{L}(f)=x$. It follows that $\varphi_{L}\left(\Gamma_{L}\right)$ is $c$, which is not mediated in $c$.

Theorem 4.28 (a) If $\varphi(\Gamma)$ is splitting in $E$, then so is $\varphi_{L}\left(\Gamma_{L}\right)$.

(b) If $\varphi(\Gamma)$ is mediated in $E$, then so is $\varphi_{V}\left(\Gamma_{V}\right)$.

Proof (a) Suppose $a_{n} \in \varphi_{L}\left(\Gamma_{L}\right)^{+}$for $n \in \mathbb{N}$ and $\sum_{n} a_{n}$ exists. Let $A \subset \mathbb{N}$. For all $n \in \mathbb{N}$ there exist $b_{n 1}, b_{n 2}, \ldots \in \varphi(\Gamma)^{+}$with $a_{n}=\sum_{m} b_{n m}$. Hence $\sum_{n} a_{n}=$ $\sum_{n, m} b_{n m}$ and so $\sum_{n, m} \mathbb{1}_{A \times \mathbb{N}}(n, m) b_{n m}=\sum_{n} \mathbb{1}_{A}(n) a_{n}$ exists in $E$.

(b) Suppose $A, B \subset \varphi_{V}\left(\Gamma_{V}\right)$ are countable sets with inf $A-B=0$. For all $a \in A$ and $b \in B$ there exist countable sets $\Upsilon_{a}, \Lambda_{b} \subset \Gamma$ with $a=\inf \varphi\left(\Upsilon_{a}\right), b=\sup \varphi\left(\Lambda_{b}\right)$. Then $\inf \varphi\left(\bigcup_{a \in A} \Upsilon_{a}-\bigcup_{b \in B} \Lambda_{b}\right)=0$ and thus inf $A=\inf \varphi\left(\bigcup_{a \in A} \Upsilon_{a}\right)=$ $\sup \varphi\left(\bigcup_{b \in B} \Lambda_{b}\right)=\sup B$.

4.29 For a Riesz space $F$ we will now investigate under which conditions the space $\Gamma_{L}$ is a Riesz subspace of $F^{X}$. The next example shows that even if $E$ is a Riesz space and $\Gamma$ is a Riesz subspace of $F^{X}, \Gamma_{L}$ may not be one. However, see Theorem 4.32.

Example 4.30 Let $a, b$ be as in Example 4.19; this time put $d=\left(0, a_{1}+a_{2}, 0, a_{3}+\right.$ $\left.a_{4}, \ldots\right)$. Then $a, d \in \Gamma_{L}$ but $a \wedge d=b \notin \Gamma_{L}$.

Hence, in Example 4.4, if $F$ is a Riesz space and $E$ is not splitting in $F$, then $\Gamma_{L}$ is not a Riesz subspace of $F^{X}$. As we will see in Theorem 4.32, considering the situation of Example 4.4: $\Gamma_{L}$ is a Riesz subspace of $F^{X}$ if and only if $E$ is splitting in $F$.

Lemma 4.31 Let $f: X \rightarrow F$ be partially in $\Gamma$.

(a) If $f$ is in $\Gamma_{L V}$, then $f \in \Gamma_{L}$.

(b) Suppose $\varphi(\Gamma)$ is splitting in E. If $g \leq f \leq h$ for certain $g, h \in \Gamma_{L}$, then $f \in \Gamma_{L}$.

Proof (a) By the definition of $\Gamma_{L V}$ there exists a $\rho \in \Gamma_{L}$ with $\rho \leq f$. Then $f-\rho$ is partially in $\Gamma, f-\rho \in \Gamma_{L V}$, and we are done if $f-\rho \in \Gamma_{L}$. Hence we may assume $f \geq 0$.

Let $\left(A_{n}\right)_{n \in \mathbb{N}}$ be a partition for $f$; we prove $\sum_{n} \varphi\left(f \mathbb{1}_{A_{n}}\right)=\varphi_{L V}(f)$. It will be clear that $\sum_{n=1}^{N} \varphi\left(f \mathbb{1}_{A_{n}}\right) \leq \varphi_{L V}(f)$ for $N \in \mathbb{N}$. For the reverse inequality let $h \in E$ be an upper bound for $\left\{\sum_{n=1}^{N} \varphi\left(f \mathbb{1}_{A_{n}}\right): N \in \mathbb{N}\right\}$. It suffices to show that $h$ must be an upper bound for $\left\{\varphi_{L}(\sigma): \sigma \in \Gamma_{L}, \sigma \leq f\right\}$.

Take a $\sigma \in \Gamma_{L}$ with $\sigma \leq f$. If $\left(B_{n}\right)_{n \in \mathbb{N}}$ is any refinement of $\left(A_{n}\right)_{n \in \mathbb{N}}$ that is a $\varphi$ partition for $\sigma$, then for all $M \in \mathbb{N}$ there exists an $N \in \mathbb{N}$ with $B_{1} \cup \ldots \cup B_{M} \subset A_{1} \cup$ $\cdots \cup A_{N}$, so that $h \geq \sum_{n=1}^{N} \varphi\left(f \mathbb{1}_{A_{n}}\right) \geq \sum_{m=1}^{M} \varphi\left(f \mathbb{1}_{B_{m}}\right) \geq \sum_{m=1}^{M} \varphi\left(\sigma \mathbb{1}_{B_{m}}\right)$. It follows from Lemma 4.17 , applied to $\sigma$, that the partition $\left(B_{m}\right)_{m \in \mathbb{N}}$ can be chosen so that this implies $h \geq \varphi_{L}(\sigma)$.

(b) As $h-g \in \Gamma_{L}$ and $0 \leq f-g \leq h-g$, we may (and do) assume $g=0$. Let $\left(A_{n}\right)_{n \in \mathbb{N}}$ be a partition for $f$ that is also a $\varphi$-partition for $h$. Now just apply (35) to

$$
a_{n}:=\varphi\left(f \mathbb{1}_{A_{n}}\right), \quad b_{n}:=\varphi\left(h \mathbb{1}_{A_{n}}\right) \quad(n \in \mathbb{N}) .
$$


As a consequence of Lemma 4.31:

Theorem 4.32 Let $F$ be a Riesz space and $\Gamma$ be a Riesz subspace of $F^{X}$. The functions $X \rightarrow F$ that are partially in $\Gamma$ form a Riesz space, $\Xi$. If $\varphi(\Gamma)$ is splitting in $E$, then $\Gamma_{L}$ is a Riesz ideal in $\Xi$, in particular, $\Gamma_{L}$ is a Riesz space.

In the classical integration theory and the Bochner integration theory one starts with considering a measure space $(X, \mathcal{A}, \mu)$ and simple functions on $X$ with values in $\mathbb{R}$ or in a Banach space. One defines an integral on these simple functions using the measure and extends this integral to a larger class of integrable functions. In 4.33 we will follow a similar procedure, replacing $\mathbb{R}$ or the Banach space with $E$ and applying the lateral extension. In Sect. 8 we will treat such extensions in more detail.

4.33 Suppose $(X, \mathcal{A}, \mu)$ is a $\sigma$-finite complete measure space and suppose $E$ is directed. Let $F=E$. For $\mathcal{I}$ we choose $\{A \in \mathcal{A}: \mu(A)<\infty\}$. The $\sigma$-finiteness of $\mu$ guarantees the existence of a partition (and vice versa).

We say that a function $f: X \rightarrow E$ is simple if there exist $N \in \mathbb{N}, a_{1}, \ldots, a_{N} \in E$, $A_{1}, \ldots, A_{N} \in \mathcal{I}$ for which

$$
f=\sum_{n=1}^{N} a_{n} \mathbb{1}_{A_{n}} .
$$

The simple functions form a stable directed linear subspace $S$ of $E^{X}$, which is a Riesz subspace of $E^{X}$ in case $E$ is a Riesz space.

For a given $f$ in $S$ one can choose a representation (40) in which the sets $A_{1}, \ldots, A_{N}$ are pairwise disjoint; thanks to the $\sigma$-finiteness of $\mu$ one can choose them in such a way that they occur in a partition $\left(A_{n}\right)_{n \in \mathbb{N}}$.

This $S$ is going to be our $\Gamma$. We define $\varphi: S \rightarrow E$ by

$$
\varphi(f)=\sum_{n=1}^{N} \mu\left(A_{n}\right) a_{n}
$$

where $f, N, A_{n}, a_{n}$ are as in (40). The $\sigma$-additivity of $\mu$ is (necessary and) sufficient to show that $S$ is laterally extendable.

A function $f: X \rightarrow E$ is partially in $S$ if and only if there exist a partition $\left(A_{n}\right)_{n \in \mathbb{N}}$ and a sequence $\left(a_{n}\right)_{n \in \mathbb{N}}$ in $E$ for which

$$
f=\sum_{n \in \mathbb{N}} a_{n} \mathbb{1}_{A_{n}} .
$$

An $f$ as in (42) with $f \geq 0$ that is partially in $S$ is an element of $S_{L}$ if and only if $\sum_{n} \mu\left(A_{n}\right) a_{n}$ exists in $E$. (See Theorem 4.9.)

\section{Combining vertical and lateral extensions}

In this section $\mathbf{E}, \mathbf{F}, \mathbf{X}, \mathcal{I}, \Gamma, \varphi$ are as in Sect. 4 . 
As we have seen, the lateral extension differs from the vertical extension in the sense that the vertical extensions of $\Gamma$ and $\varphi$ can always be made, but for lateral extension we had to assume the space $\Gamma$ to be stable and $\varphi$ to be laterally extendable (see 4.11). In this section we investigate when one can make a lateral extension of another (say vertical) extension. Furthermore we will compare different extensions and combinations of extensions.

Instead of $\left(\Gamma_{L}\right)_{V}$ and $\left(\left(\Gamma_{L}\right)_{V}\right)_{L}$ we write $\Gamma_{L V}$ and $\Gamma_{L V L} ;$ similarly $\varphi_{L V}=\left(\varphi_{L}\right)_{V}$ etc.

5.1 By Theorem 4.18 the following holds for a stable directed linear subspace $\Delta$ of $F^{X}$ and a laterally extendable order preserving linear map $\omega: \Delta \rightarrow E:$ If $\Delta_{L}$ is stable, then $\omega_{L}$ is laterally extendable (and so $\Delta_{L L}$ exists). If $\Delta_{V}$ is stable, then $\omega_{V}$ is laterally extendable (and so $\Delta_{V L}$ exists). We will use these facts without explicit mention.

5.2 The following statements follow from the definitions and theorems we have:

(a) $\Gamma_{V} \subset \Gamma_{L V}$ and $\varphi_{L V}=\varphi_{V}$ on $\Gamma_{V}$.

(b) $\Gamma_{L} \subset \Gamma_{L V}$ and $\varphi_{L V}=\varphi_{L}$ on $\Gamma_{L}$.

(c) $\varphi_{V}=\varphi_{L}$ on $\Gamma_{L} \cap \Gamma_{V}$.

For (d), (e) and (f) let $\Gamma_{V}$ be stable.

(d) $\Gamma_{L V} \subset \Gamma_{V L V}$ and $\varphi_{V L V}=\varphi_{L V}$ on $\Gamma_{L V}$.

(e) $\Gamma_{V L} \subset \Gamma_{V L V}$ and $\varphi_{V L V}=\varphi_{V L}$ on $\Gamma_{V L}$.

(f) $\varphi_{L V}=\varphi_{V L}$ on $\Gamma_{L V} \cap \Gamma_{V L}$.

Observe that as a consequence of (a) and (b): if $f \in \Gamma_{L}$ and $g \in \Gamma_{V}$ and $f \leq g$ (or $f \geq g$ ), then $\varphi_{L}(f) \leq \varphi_{V}(g)$ (or $\varphi_{L}(f) \geq \varphi_{V}(g)$ ). Moreover, as a consequence of (c) and (d); if $\Gamma_{V}$ is stable: if $f \in \Gamma_{L V}$ and $g \in \Gamma_{V L V}$ and $f \leq g$ (or $f \geq g$ ), then $\varphi_{L V}(f) \leq \varphi_{V L V}(g)\left(\right.$ or $\left.\varphi_{L V}(f) \geq \varphi_{V L V}(g)\right)$.

5.3 Note that if $\Gamma$ is stable and $\varphi$ is laterally extendable, then we can extend $\Gamma$ to $\Gamma_{V}, \Gamma_{L}$ and $\Gamma_{L V}$. If, moreover, $\Gamma_{V}$ is stable, then we can also extend $\Gamma$ to $\Gamma_{V L}$ and $\Gamma_{V L V}$. However, "more stability" will not give us larger extensions than $\Gamma_{V L V}$. Indeed, if $\Gamma_{L V}$ is stable then $\Gamma_{L V} \subset \Gamma_{L V L}=\Gamma_{V L}$ (see Theorem 5.8). If moreover $\Gamma_{V L V}$ is stable, then even $\Gamma_{V L V L}=\Gamma_{V L V}=\Gamma_{V L}$.

Lemma 5.4 (a) If $f \in \Gamma_{L V}^{+}$, then there exists a countable $\Lambda \subset \Gamma$ with $\Lambda \leq f$ and $\varphi_{L V}(f)=\sup \varphi(\Lambda)$.

(b) If $\Gamma_{V}$ is stable and $f \in \Gamma_{V L}^{+}$, then there exists a countable $\Lambda \subset \Gamma$ with $\Lambda \leq f$ and $\varphi_{V L}(f)=\sup \varphi(\Lambda)$.

Proof (a) There exist $\sigma_{1}, \sigma_{2}, \ldots$ in $\Gamma_{L}$ with $\sigma_{n} \leq f$ for all $n \in \mathbb{N}$ and $\sup _{n \in \mathbb{N}} \varphi_{L}\left(\sigma_{n}\right)=\varphi_{L V}(f)$. Hence, we are done if for every $\sigma$ in $\Gamma_{L}$ with $\sigma \leq f$ there is a countable set $\Lambda_{\sigma} \subset\{\rho \in \Gamma: \rho \leq f\}$ such that every upper bound for $\varphi\left(\Lambda_{\sigma}\right)$ majorizes $\varphi_{L}(\sigma)$. But that is not hard to prove. For such a $\sigma$, by Lemma 4.17 there exists a partition $\left(B_{m}\right)_{m \in \mathbb{N}}$ for which (32) holds. Now let $\Lambda_{\sigma}$ be $\left\{\sum_{m=1}^{M} \sigma \mathbb{1}_{B_{m}}: M \in \mathbb{N}\right\}$.

(b) Suppose $\Gamma_{V}$ is stable. Let $\left(A_{n}\right)_{n \in \mathbb{N}}$ be a $\varphi_{V}$-partition for $f$. Then the set $\Lambda_{f}=$ $\left\{\sum_{n=1}^{N} f \mathbb{1}_{A_{n}}: N \in \mathbb{N}\right\}$ is a countable subset of $\Gamma_{V}$ and $\sup \varphi_{V}\left(\Lambda_{f}\right)=\varphi_{V L}(f)$. 
Moreover, for every $N \in \mathbb{N}$ there is a countable set $\Lambda_{N} \subset\{\sigma \in \Gamma: \sigma \leq$ $\left.\sum_{n=1}^{N} f \mathbb{1}_{A_{n}}\right\}$ for which $\sup \varphi\left(\Lambda_{N}\right)=\varphi_{V}\left(\sum_{n=1}^{N} f \mathbb{1}_{A_{n}}\right)$. Take $\Lambda=\bigcup_{N \in \mathbb{N}} \Lambda_{N}$.

Theorem 5.5 For (b), (c), (d) and (e) let $\Gamma_{V}$ be stable and $f$ be partially in $\Gamma_{V}$.

(a) If $f \in \Gamma_{L V}$, then 6

$$
f \in \Gamma_{V} \Longleftrightarrow \text { there exist } \pi, \rho \in \Gamma \text { with } \pi \leq f \leq \rho \text {. }
$$

(b) If $f \in \Gamma_{V L}$, then

$$
f \in \Gamma_{V} \Longleftrightarrow \text { there exist } \pi, \rho \in \Gamma \text { with } \pi \leq f \leq \rho^{6} .
$$

(c) $f \in \Gamma_{L V} \Longleftrightarrow f \in \Gamma_{V L}$ and there exist $\pi, \rho \in \Gamma_{L}$ with $\pi \leq f \leq \rho$.

(d) If $\varphi_{V}\left(\Gamma_{V}\right)$ is splitting in $E$, then

$$
f \in \Gamma_{V L} \Longleftrightarrow \text { there exist } \pi, \rho \in \Gamma_{V L} \text { with } \pi \leq f \leq \rho \text {. }
$$

(e) If $\varphi_{V}\left(\Gamma_{V}\right)$ is splitting in $E$, then

$$
f \in \Gamma_{V L} \cap \Gamma_{L V} \Longleftrightarrow \text { there exist } \pi, \rho \in \Gamma_{L} \text { with } \pi \leq f \leq \rho .
$$

Proof The proofs of (a) and (b) are similar to the proof of (c) and therefore omitted.

(c) $\Leftarrow$ : By Lemma 5.4 (b) there exist countable sets $\Lambda, \Upsilon \subset \Gamma$ with $\Lambda \leq f-\pi$ and $\Upsilon \leq \rho-f$ for which $\sup \varphi(\Lambda)=\varphi_{V L}(f-\pi)$ and $\sup \varphi(\Upsilon)=\varphi_{V L}(\rho-f)$. Then $\Lambda+\pi$ and $\rho-\Upsilon$ are countable subsets of $\Gamma_{L}$ with $\Lambda+\pi \leq f \leq \rho-\Upsilon$ and $\sup \varphi_{L}(\Lambda+\pi)=\varphi_{V L}(f)=\inf \varphi_{L}(\rho-\Upsilon)$. Hence $f \in \Gamma_{L V}$.

$\Rightarrow$ : Let $f \in \Gamma_{L V}$ and be partially in $\Gamma_{V}$. There exists a $\pi \in \Gamma_{L}$ for which $f-\pi \in \Gamma_{L V}^{+}$, hence we may assume $f \geq 0$. Let $\left(A_{n}\right)_{n \in \mathbb{N}}$ be a $\Gamma_{V}$-partition for $f$, i.e., $f \mathbb{1}_{A_{n}} \in \Gamma_{V}$ and thus $\varphi_{L V}\left(f \mathbb{1}_{A_{n}}\right)=\varphi_{V}\left(f \mathbb{1}_{A_{n}}\right)$ for all $n \in \mathbb{N}$ (see 5.2(a)). Then $\varphi_{L V}(f) \geq \sum_{n=1}^{N} \varphi_{V}\left(f \mathbb{1}_{A_{n}}\right)$ for all $N \in \mathbb{N}$. Let $h \in E$ be such that $h \geq \sum_{n=1}^{N} \varphi_{V}\left(f \mathbb{1}_{A_{n}}\right)$ for all $N \in \mathbb{N}$. From Lemma 4.17 we infer that $h \geq \varphi_{L}(\sigma)$ for every $\sigma \in \Gamma_{L}$ with $\sigma \leq f$. We conclude that $\sum_{n} \varphi_{V}\left(f \mathbb{1}_{A_{n}}\right)=\varphi_{L V}(f)$, i.e., $f \in \Gamma_{V L}$.

(d) $\Leftarrow$ : We may assume $\pi=0$. Let $\left(A_{n}\right)_{n \in \mathbb{N}}$ be a $\varphi_{V}$-partition for $\rho$ with $f \mathbb{1}_{A_{n}} \in \Gamma_{V}$ for all $n \in \mathbb{N}$. Then $0 \leq \varphi_{V}\left(f \mathbb{1}_{A_{n}}\right) \leq \varphi_{V}\left(\rho \mathbb{1}_{A_{n}}\right)$ for all $n \in \mathbb{N}$ and $\sum_{n} \varphi_{V}\left(\rho \mathbb{1}_{A_{n}}\right)$ exists in $E$. Hence, so does $\sum_{n} \varphi_{V}\left(f \mathbb{1}_{A_{n}}\right)$, i.e., $f \in \Gamma_{V L}$.

(e) is a consequence of (c) and (d).

In the following example all functions in $\Gamma_{L V}$ are partially in $\Gamma_{V}$.

\footnotetext{
6 By the definition of ideal in [9] or [10] (note that $\Gamma_{V}$ is directed) this means that $\Gamma_{V}$ is the smallest ideal in $\Gamma_{L V}$ (and for (b); in $\Gamma_{V L}$ ) that contains $\Gamma$.
} 
Example 5.6 Consider $X=\mathbb{N}, \mathcal{I}=\mathcal{P}(\mathbb{N}), E=F$; let $D$ be a linear subspace of $E$ and let $D_{V}$ be the vertical extension of $D$ with respect to the inclusion map $D \rightarrow E$. Let $\Gamma=c_{00}[D]$ and $\varphi: \Gamma \rightarrow E$ be $\varphi(f)=\sum_{n \in \mathbb{N}} f(n)$. Then $\Gamma_{V}=c_{00}\left[D_{V}\right]$. Let $f \in \Gamma_{L V}$. We will show that $f(k) \in D_{V}$ and thus that $f$ is partially in $\Gamma_{V}$. Let $\sigma_{n}, \tau_{n} \in \Gamma_{L}$ be such that $\sigma_{n} \leq f \leq \tau_{n}$ and $\inf _{n \in \mathbb{N}} \varphi\left(\tau_{n}\right)=\sup _{n \in \mathbb{N}} \varphi\left(\sigma_{n}\right)$. Then $\inf _{n \in \mathbb{N}}\left(\tau_{n}(k)-\sigma_{n}(k)\right) \leq \inf _{n \in \mathbb{N}} \varphi\left(\tau_{n}-\sigma_{n}\right)=0$. Since $\sigma_{n}(k), \tau_{n}(k) \in D$ for all $n \in \mathbb{N}$, we have $f(k) \in D_{V}$.

Thus every $f \in \Gamma_{L V}$ is partially in $\Gamma_{V}$. Since $\Gamma_{V}$ is stable, by Theorem 5.5(c) we conclude that $\Gamma_{L V} \subset \Gamma_{V L}$.

Lemma 5.7 Suppose that $\Gamma_{L V}$ is stable. Then every $f \in \Gamma_{L V}$ is partially in $\Gamma_{V}$.

Proof Let $f \in \Gamma_{L V}$ and let $\pi, \rho \in \Gamma_{L}$ be such that $\pi \leq f \leq \rho$. Let $\left(A_{n}\right)_{n \in \mathbb{N}}$ be a $\varphi$-partition for both $\pi$ and $\rho$. Then $f \mathbb{1}_{A_{n}} \in \Gamma_{L V}$ and $\pi \mathbb{1}_{A_{n}} \leq f \mathbb{1}_{A_{n}} \leq \rho \mathbb{1}_{A_{n}}$ for all $n \in \mathbb{N}$. By Theorem 5.5(a) we conclude that $f \mathbb{1}_{A_{n}} \in \Gamma_{V}$.

Theorem 5.8 Suppose that $\Gamma_{V}$ and $\Gamma_{L V}$ are stable. Then $\Gamma_{L V} \subset \Gamma_{V L}=\Gamma_{L V L}$. Write $\bar{\Gamma}=\Gamma_{V L}$ and $\bar{\varphi}=\varphi_{V L}$. If $\bar{\Gamma}$ is stable, then $\bar{\Gamma}_{L}=\bar{\Gamma}$ and $\bar{\varphi}_{L}=\bar{\varphi}$. If $\bar{\Gamma}_{V}$ is stable, then $\bar{\Gamma}_{V}=\bar{\Gamma}$ and $\bar{\varphi}_{V}=\bar{\varphi}$.

In particular, if $\varphi_{L}\left(\Gamma_{L}\right)$ is mediated in $E$ and $\varphi_{V}\left(\Gamma_{V}\right)$ is splitting in $E$, then $\Gamma_{V}$, $\Gamma_{L V}$ and $\Gamma_{V L}$ are stable (see Theorem 4.25) and thus $\Gamma_{L V} \subset \bar{\Gamma}, \bar{\Gamma}=\bar{\Gamma}_{V}=\bar{\Gamma}_{L}$, $\bar{\varphi}=\bar{\varphi}_{V}=\bar{\varphi}_{L}$, so $\overline{\bar{\Gamma}}=\bar{\Gamma}($ and $\overline{\bar{\varphi}}=\bar{\varphi})$.

Proof The inclusion $\Gamma_{L V} \subset \Gamma_{V L}$ follows by Theorem 5.5(c) and Lemma 5.7. We prove $\Gamma_{L V L} \subset \Gamma_{V L}$. For $f \in \Gamma_{L V L}^{+}$there is a $\varphi_{L V}$-partition for $f$ and since $\Gamma_{L V} \subset$ $\Gamma_{V L}$ this is also a $\varphi_{V L}$-partition for $f$, hence there exists a $\varphi_{V}$-partition for $f$, i.e., $f \in \Gamma_{V L}$.

Suppose $\bar{\Gamma}$ is stable. Then $\bar{\Gamma}_{L}=\left(\Gamma_{V L}\right)_{L}=\Gamma_{V L}=\bar{\Gamma}$ and $\bar{\varphi}_{L}=\bar{\varphi}$ by Theorem 4.18(a).

Suppose $\bar{\Gamma}_{V}$ to be stable. As $\Gamma_{V}$ is stable we can apply the first part of the theorem to $\Gamma_{V}$ instead of $\Gamma$. Indeed, $\left(\Gamma_{V}\right)_{V}$ and $\left(\Gamma_{V}\right)_{L V}$ are stable, since $\left(\Gamma_{V}\right)_{V}=\Gamma_{V}$ and $\left(\Gamma_{V}\right)_{L V}=\bar{\Gamma}_{V}$. Hence, $\left(\Gamma_{V}\right)_{L V} \subset\left(\Gamma_{V}\right)_{V L}=\Gamma_{V L}$, i.e., $\bar{\Gamma}_{V} \subset \bar{\Gamma}$ (and $\left.\bar{\varphi}_{V}=\bar{\varphi}\right)$.

Suppose $\varphi_{L}\left(\Gamma_{L}\right)$ is mediated in $E$ and $\varphi_{V}\left(\Gamma_{V}\right)$ is splitting in $E$. Then $\Gamma_{L}, \Gamma_{V}$ and $\Gamma_{L V}$ are stable by Theorem 4.25(a),(b) and (c). Consequently, again by Theorem 4.25(b) $\Gamma_{V L}$ is stable.

Corollary 5.9 Suppose $E$ is mediated (and thus splitting), $\bar{\Gamma}=\Gamma_{V L}$. Then $\bar{\Gamma}=$ $\bar{\Gamma}_{V}=\bar{\Gamma}_{L}$, so $\overline{\bar{\Gamma}}=\bar{\Gamma}($ and $\overline{\bar{\varphi}}=\bar{\varphi})$.

At the end of Sect. 5 we will show that sometimes $\Gamma_{V L} \subsetneq \Gamma_{L V}$ (Example 5.14) and sometimes $\Gamma_{L V} \subsetneq \Gamma_{V L}$ (Example 5.15). Note that this implies that $\Gamma_{V L V}$ can be strictly larger then either $\Gamma_{V L}$ or $\Gamma_{L V}$.

Theorem 5.8 raises the question whether stability of $\Gamma_{V}$ entails $\Gamma_{V L} \subset \Gamma_{L V}$. In general the answer is negative; see Example 5.15. In Theorem 5.10 we give conditions sufficient for the inclusion.

Theorem 5.10 Suppose $\Gamma_{V}$ is stable. Consider these two statements.

(a) For every $f \in \Gamma_{V L}^{+}$there is a $\rho$ in $\Gamma_{L}^{+}$with $f \leq \rho$. 
(b) E satisfies:

If $Y_{1}, Y_{2}, \ldots \subset E$ are nonempty countable with inf $Y_{n}=0$ for all $n \in \mathbb{N}$, then there exist $y_{1} \in Y_{1}, y_{2} \in Y_{2}, \ldots$ such that $\sum_{n} y_{n}$ exists in $E$.

If (a) is satisfied, then $\Gamma_{V L} \subset \Gamma_{L V}$. (b) implies (a).

Proof If (a) is satisfied, then by Theorem 5.5(c) follows that $\Gamma_{V L} \subset \Gamma_{L V}$.

Suppose (b). Let $f \in \Gamma_{V L}^{+}$. Let $\left(A_{n}\right)_{n \in \mathbb{N}}$ be a $\varphi_{V}$-partition for $f$. For $n \in \mathbb{N}$, let $\Upsilon_{n} \subset \Gamma$ be a countable set with $f \mathbb{1}_{A_{n}} \leq \Upsilon_{n}$ and

$$
\varphi_{V}\left(f \mathbb{1}_{A_{n}}\right)=\inf \varphi\left(\Upsilon_{n}\right)
$$

We may assume $\sigma \mathbb{1}_{A_{n}}=\sigma$ for all $\sigma \in \Upsilon_{n}$. Choose $\sigma_{n} \in \Upsilon_{n}$ for $n \in \mathbb{N}$ such that $\sum_{n}\left(\varphi\left(\sigma_{n}\right)-\varphi_{V}\left(f \mathbb{1}_{A_{n}}\right)\right)$ and thus $\sum_{n} \varphi\left(\sigma_{n}\right)$ exist in $E$. Then $\rho:=\sum_{n \in \mathbb{N}} \sigma_{n}$ is in $\Gamma_{L}^{+}$ with $f \leq \rho$.

5.11 We will discuss examples of spaces $E$ for which (43) holds.

(I) If $E$ is a Banach lattice with $\sigma$-order continuous norm, then $E$ satisfies (43) (one can find $y_{n} \in Y_{n}$ with $\left.\left\|y_{n}\right\| \leq 2^{-n}\right)$.

(II) Let $(X, \mathcal{A}, \mu)$ be a complete $\sigma$-finite measure space and assume there exists a $g \in$ $L^{1}(\mu)$ with $g>0 \mu$-a.e. Then the space $E$ of equivalence classes of measurable functions $X \rightarrow \mathbb{R}$ satisfies (43): It is sufficient to prove that if $Z_{1}, Z_{2}, \ldots \subset E$ are nonempty countable with $\inf Z_{n}=0$ for all $n \in \mathbb{N}$, then there exists $z_{1} \in$ $Z_{1}, z_{2} \in Z_{2}, \ldots$ and a $z \in E$ such that $z_{n} \leq z$ for all $n \in \mathbb{N}$ (for $Z_{n}$ take $\left.2^{n} Y_{n}\right)$. One can prove that such a $z$ exists by mapping the equivalence classes of measurable functions into $L^{1}(\mu)$ by the order isomorphism $f \mapsto(\arctan \circ f) g$.

(III) $\mathbb{R}^{\mathbb{N}}$ is a special case of (II), therefore satisfies (43).

Theorem 5.12 Let E be mediated and splitting and satisfy (43) (e.g. E be a Banach lattice with $\sigma$-order continuous norm (Theorem 4.24), or $E$ is the space mentioned in 5.11(II)). Then $\Gamma_{V}$ is stable and $\Gamma_{V L}=\Gamma_{L V}, \varphi_{V L}=\varphi_{L V}$.

Proof This is a consequence of Theorems 5.8 and 5.10.

For a Riesz space $F$ and a Riesz subspace $\Gamma$ of $F^{X}$ we will now investigate under which conditions on $\varphi(\Gamma), \varphi_{L}\left(\Gamma_{L}\right)$ and $\varphi_{V}\left(\Gamma_{V}\right)$ the spaces $\Gamma_{L V}$ and $\Gamma_{V L}$ are Riesz subspaces of $F^{X}$.

Theorem 5.13 Suppose $F$ is a Riesz space and $\Gamma$ is a Riesz subspace of $F^{X}$. If $\varphi(\Gamma)$ is splitting in $E$ and $\varphi_{L}\left(\Gamma_{L}\right)$ is mediated in $E$, then $\Gamma_{L V}$ is a Riesz subspace of $F^{X}$. If $\varphi(\Gamma)$ is mediated in $E$ and $\varphi_{V}\left(\Gamma_{V}\right)$ is splitting in $E$, then $\Gamma_{V L}$ is a Riesz subspace of $F^{X}$.

In particular, if $E$ is mediated (and thus splitting), then both $\Gamma_{L V}$ and $\Gamma_{V L}$ are Riesz subspaces of $F^{X}$. 
Proof Note first that if $\varphi(\Gamma)$ is mediated in $E$, then $\Gamma_{V}$ is stable by Theorem 4.25(b). For a proof, combine Theorem 4.32 and Corollary 3.10.

The next example illustrates that $\Gamma_{L V}$ is not always included in $\Gamma_{V L}$ (given that $\Gamma_{V}$ is stable) even if $E$ and $F$ are Riesz spaces and $\Gamma, \Gamma_{L V}, \Gamma_{V L}$ Riesz subspaces of $F^{X}$.

Example $5.14\left[\Gamma_{V L} \subsetneq \Gamma_{L V}=\Gamma_{V L V}\right]$ For an element $b=\left(\beta_{1}, \beta_{2}, \ldots\right)$ of $\mathbb{R}^{\mathbb{N}}$ we write $b=\sum_{n \in \mathbb{N}} \beta_{n} e_{n}$.

Consider $X=\{0,1,2, \ldots\}$ and $\mathcal{I}=\mathcal{P}(X)$. Let $E=c, F=\mathbb{R}^{\mathbb{N}}, \Omega=F^{X}$. We view the elements of $\Omega$ as sequences $\left(a, b_{1}, b_{2}, \ldots\right)$ with $a, b_{1}, b_{2}, \ldots \in \mathbb{R}^{\mathbb{N}}$.

Define sets $\Gamma \subset \Theta \subset \Omega$ and a map $\Phi: \Theta \rightarrow \mathbb{R}^{\mathbb{N}}$ by

$$
\begin{aligned}
& \Theta=\left\{\left(a, \beta_{1} e_{1}, \beta_{2} e_{2}, \ldots\right): a \in c, \beta_{1}, \beta_{2}, \ldots \in \mathbb{R}\right\}, \\
& \Phi\left(a, \beta_{1} e_{1}, \beta_{2} e_{2}, \ldots\right)=a+\sum_{n \in \mathbb{N}} \beta_{n} e_{n} \quad\left(a \in c, \beta_{1}, \beta_{2}, \ldots \in \mathbb{R}\right), \\
& \Gamma=\left\{\left(a, \beta_{1} e_{1}, \beta_{2} e_{2}, \ldots\right): a \in c,\left(\beta_{1}, \beta_{2}, \ldots\right) \in c_{00}\right\} .
\end{aligned}
$$

Then $\Phi(\Gamma)=c=E$; let $\varphi=\left.\Phi\right|_{\Gamma}$. From the definition it is easy to see that $\Gamma$ is stable and $\varphi$ is laterally extendable. We leave it to the reader to verify that $\Gamma_{V}=\Gamma$,

$$
\Gamma_{L}=\left\{\left(a, \beta_{1} e_{1}, \beta_{2} e_{2}, \ldots\right): a \in c,\left(\beta_{1}, \beta_{2}, \ldots\right) \in c\right\}
$$

and $\varphi_{L}=\Phi$ on $\Gamma_{L}$.

It follows that $\Gamma_{V}$ is stable and $\Gamma_{V L}=\Gamma_{L} \subset \Gamma_{L V}=\Gamma_{V L V}$. We prove $\Gamma_{V L} \neq \Gamma_{L V}$. To this end, define $h \in \Omega$ by

$$
\left\{\begin{array}{l}
h(n)=(-1)^{n} e_{n} \quad(n=1,2, \ldots), \\
h(0)=-\sum_{n \in \mathbb{N}} h(n)=-\sum_{n \in \mathbb{N}}(-1)^{n} e_{n} .
\end{array}\right.
$$

As $h(0) \notin c$ we have $h \mathbb{1}_{\{0\}} \notin \Gamma$; in particular, $h$ is not partially in $\Gamma$, so $h \notin \Gamma_{L}=\Gamma_{V L}$. It remains to prove $h \in \Gamma_{L V}$.

For $k \in \mathbb{N}$, define $\tau_{k}, \sigma_{k}: X \rightarrow \mathbb{R}^{\mathbb{N}}$ :

$$
\begin{aligned}
& \begin{cases}\tau_{k}(0)=-\sum_{n=1}^{k}(-1)^{n} e_{n}+\sum_{n=k+1}^{\infty} e_{n}, & (n=1, \ldots, k), \\
\tau_{k}(n)=h(n)=(-1)^{n} e_{n} & (n=k+1, k+2, \ldots), \\
\tau_{k}(n)=e_{n} & \end{cases} \\
& \begin{cases}\sigma_{k}(0)=-\sum_{n=1}^{k}(-1)^{n} e_{n}-\sum_{n=k+1}^{\infty} e_{n}, & \\
\sigma_{k}(n)=h(n)=(-1)^{n} e_{n} & (n=1, \ldots, k), \\
\sigma_{k}(n)=-e_{n} & \end{cases}
\end{aligned}
$$

Then $\tau_{k}, \sigma_{k} \in \Gamma_{L}, \tau_{k} \geq h \geq \sigma_{k}, \varphi_{L}\left(\tau_{k}\right)=\Phi\left(\tau_{k}\right)=2 \sum_{n>k} e_{n}, \varphi_{L}\left(\sigma_{k}\right)=$ $-2 \sum_{n>k} e_{n}, \operatorname{so~inf}_{k \in \mathbb{N}} \varphi_{L}\left(\tau_{k}\right)=\sup _{k \in \mathbb{N}} \varphi_{L}\left(\sigma_{k}\right)=0$, and $h \in \Gamma_{L V}$. 



Fig. 1 Graph of $f_{n}$

The next example illustrates that $\Gamma_{V L}$ is not always included in $\Gamma_{L V}$; it provides an example of an $f \in \Gamma_{V L}^{+}$for which there exist no $\rho \in \Gamma_{L}^{+}$with $f \leq \rho$ (see Theorem $5.5(\mathrm{c}))$.

Example 5.15 $\left[\Gamma_{L V} \subsetneq \Gamma_{V L}\right]$ Let $E=C[0,1]$ and let $D \subset C[0,1]$ be the set of polynomials of degree $\leq 2$. The set $D$ is order dense ${ }^{7}$ in $C[0,1]$ (see [11, Example4.4]). Hence, for all $f \in E$ there exist $\left(g_{n}\right)_{n \in \mathbb{N}},\left(h_{n}\right)_{n \in \mathbb{N}}$ in $D$ with $f=\inf _{n \in \mathbb{N}} g_{n}=$ $\sup _{n \in \mathbb{N}} h_{n}$. Therefore $E$ is the vertical extension of $D$ with respect to the inclusion map $D \rightarrow E$.

Take $X=\mathbb{N}, \mathcal{I}=\mathcal{P}(\mathbb{N}), F=E=C[0,1], \Gamma=c_{00}[D] \subset F^{\mathbb{N}}=E^{\mathbb{N}}$ and let $\varphi: \Gamma \rightarrow E$ be given by $\varphi(f)=\sum_{n \in \mathbb{N}} f(n)$. Since this situation is the same as in Example 5.6 with $D_{V}=E$, we have $\Gamma_{V}=c_{00}[E]$ and $\Gamma_{L V} \subset \Gamma_{V L}$.

Furthermore (see 4.6)

$$
\begin{aligned}
\Gamma_{L}^{+} & =\left\{f \in\left(D^{+}\right)^{\mathbb{N}}: \sum_{n} f(n) \text { exists in } E\right\}, \\
\Gamma_{V L}^{+} & =\left\{f \in\left(E^{+}\right)^{\mathbb{N}}: \sum_{n} f(n) \text { exists in } E\right\} .
\end{aligned}
$$

We construct an $f \in \Gamma_{V L}^{+}$that is not in $\Gamma_{L V}$. For $n \in \mathbb{N}$ let $f_{n}$ be the 'tent' function defined by (Fig. 1)

$$
\begin{aligned}
& f_{n}(0)=0 ; \quad f_{n}\left(\frac{1}{n}\right)=1 ; \quad f_{n}\left(\frac{1}{i}\right)=0 \quad \text { if } i \in \mathbb{N}, i \neq n \\
& f_{n} \text { is affine on the interval }\left[\frac{1}{1+i}, \frac{1}{i}\right] \text { for all } i \in \mathbb{N} .
\end{aligned}
$$

Then $\sum_{n=1}^{\infty} f_{n}=\mathbb{1}_{(0,1]}$ pointwise, so $\sum_{n} f_{n}=\mathbb{1}$ in $C[0,1]$. Hence $f=$ $\left(f_{1}, f_{2}, f_{3}, \ldots\right) \in \Gamma_{V L}^{+}$.

We will prove that $f \notin \Gamma_{L V}$; by showing there exists no $\rho \in \Gamma_{L}$ for which $f \leq \rho$.

Suppose $\rho \in \Gamma_{L}$ and $f \leq \rho$. Then $\rho=\left(\rho_{1}, \rho_{2}, \ldots\right)$ where $\rho_{1}, \rho_{2}, \ldots$ are elements of $D^{+}$and $j=\sum_{n} \rho_{n}$ exists in $E=C[0,1]$. Let $M$ be the largest value of $j$. Every $\rho_{n}$ is a quadratic function that maps $[0,1]$ into $[0, M]$. Consequently (see the postscript)

$$
\left|\rho_{n}(x)-\rho_{n}(y)\right| \leq 4 M|x-y| \quad(x, y \in[0,1], n \in \mathbb{N}) .
$$

\footnotetext{
7 A subspace $D$ of a partially ordered vector space $E$ is called order dense in $E$ if $x=\sup \{d \in D: d \leq x\}$ (and thus $x=\inf \{d \in D: d \geq x\}$ ) for all $x \in E$.
} 
In particular, $\rho_{n}(0) \geq \rho_{n}\left(\frac{1}{n}\right)-4 M \frac{1}{n} \geq f_{n}\left(\frac{1}{n}\right)-4 M \frac{1}{n}=1-4 M \frac{1}{n} \geq \frac{1}{2}$ for $n \geq 8 M$. As $j(0) \geq \sum_{n \geq N} \rho_{n}(0)$ for all $N \in \mathbb{N}$, this is a contradiction.

Postscript Let $h: x \mapsto a x^{2}+b x+c$ be a quadratic function on $[0,1]$ and $0 \leq$ $h(x) \leq M$ for all $x$; we prove $\left|h^{\prime}(x)\right| \leq 4 M$ for all $x \in[0,1]$. Since the derivative is either decreasing or increasing, we have $\left|h^{\prime}(x)\right| \leq \max \left\{\left|h^{\prime}(0)\right|,\left|h^{\prime}(1)\right|\right\}$. Now $h^{\prime}(0)=$ $b=4 h\left(\frac{1}{2}\right)-h(1)-3 h(0)$ and $h^{\prime}(1)=2 a+b=3 h(1)+h(0)-4 h\left(\frac{1}{2}\right)$. Since $|h(x)-h(y)| \leq M$ for all $x, y \in[0,1]$, we get the bounds $\left|h^{\prime}(0)\right| \leq 4 M$ and $\left|h^{\prime}(1)\right| \leq 4 M$ as desired.

5.16 Observe that $\Gamma_{V L}$ in Example 5.15 is not stable since $\left(f_{1}, 0, f_{3}, 0, \ldots\right) \notin \Gamma_{V L}$.

\section{Embedding $E$ in a (slightly) larger space}

\section{In this section $\mathbf{E}, \mathbf{F}, \mathbf{X}, \mathcal{I}, \Gamma, \varphi$ are as in Sect. 4 .}

Suppose $E^{\bullet}$ is another partially ordered vector space and $E \subset E^{\bullet}$. Consider $\varphi^{\bullet}$ : $\Gamma \rightarrow E^{\bullet}$, where $\varphi^{\bullet}(f)=\varphi(f)$ for $f \in \Gamma$.

Write $\Gamma_{V}^{\bullet}$ for the vertical extension of $\Gamma$ with respect to $\varphi^{\bullet}$. If $\varphi^{\bullet}$ is laterally extendable, write $\Gamma_{L}^{\bullet}$ for the lateral extension of $\Gamma$ with respect to $\varphi^{\bullet}, \Gamma_{L V}^{\bullet}$ for the vertical extension of $\Gamma_{L}^{\bullet}$ with respect to $\varphi_{L}^{\bullet}$. Similarly, if $\Gamma_{V}^{\bullet}$ is stable, we introduce the notations $\Gamma_{V L}^{\bullet}$ and $\Gamma_{V L V}^{\bullet}$.

It is not generally the case that $\Gamma_{V} \subset \Gamma_{V}^{\bullet}$ or $\Gamma_{L} \subset \Gamma_{L}^{\bullet}$, but a natural restriction on $E^{\bullet}$ helps; see Theorem 6.2.

For $E^{\bullet}$ we can choose to be a Dedekind complete Riesz space in which countable suprema of $E$ are preserved, in case $E$ is Archimedean and directed (see 6.3). In this situation, in some sense, $\Gamma_{V L}^{\bullet}$ is the largest extension one can obtain.

Definition 6.1 Let $D$ be a subspace of a partially ordered vector space $P$. Then we say that countable suprema in $D$ are preserved in $P$ if the following implication holds for all $a \in D$ and all countable $A \subset D$

$A$ has supremum $a$ in $D \Longrightarrow A$ has supremum $a$ in $P$.

Note that the reverse implication holds always.

The following theorem is a natural consequence.

Theorem 6.2 Suppose that countable suprema in $E$ are preserved in $E^{\bullet}$. Then $\varphi^{\bullet}$ is laterally extendable and

$$
\begin{aligned}
& f \in \Gamma_{V} \Longleftrightarrow f \in \Gamma_{V}^{\bullet} \text { and } \varphi_{V}^{\bullet}(f) \in E, \\
& f \in \Gamma_{L} \Longleftrightarrow f \in \Gamma_{L}^{\bullet} \text { and } \varphi_{L}^{\bullet}(f) \in E, \\
& \varphi_{V}^{\bullet}(f)=\varphi_{V}(f) \text { for } f \in \Gamma_{V}, \quad \varphi_{L}^{\bullet}(f)=\varphi_{L}(f) \text { for } f \in \Gamma_{L}, \\
& \Gamma_{L V} \subset \Gamma_{L V}^{\bullet}, \quad \varphi_{L V}^{\bullet}(f)=\varphi_{L V}(f) \text { for } f \in \Gamma_{L V} .
\end{aligned}
$$


Suppose $\Gamma_{V}$ and $\Gamma_{V}^{\bullet}$ are stable. Then

$$
\begin{aligned}
& \Gamma_{V L} \subset \Gamma_{V L}^{\bullet}, \quad \varphi_{V L}^{\bullet}(f)=\varphi_{V L}(f) \text { for } f \in \Gamma_{V L}, \\
& \Gamma_{V L V} \subset \Gamma_{V L V}^{\bullet}, \quad \varphi_{V L V}^{\bullet}(f)=\varphi_{V L V}(f) \text { for } f \in \Gamma_{V L V} .
\end{aligned}
$$

6.3 Under the assumptions made in Sect. $4 \Gamma$ is directed, thus so are $\Gamma_{L}, \Gamma_{V}$ (see 3.11) and $\Gamma_{L V}$ (etc.). Hence $\varphi_{V}\left(\Gamma_{V}\right), \varphi_{L}\left(\Gamma_{L}\right), \varphi_{L V}\left(\Gamma_{L V}\right)$ (etc.) are all subsets of $E^{+}-E^{+}$. For this reason we may assume that $E$ itself is directed.

Then under the (rather general) assumption that $E$ is also Archimedean (see Definition 3.19), $E$ can be embedded in a Dedekind complete Riesz space such that suprema and infima in $E$ are preserved, as we state in Theorem 6.4.

Consequently, choosing such a Dedekind complete Riesz space for $E^{\bullet}$ one has the following: $\Gamma_{V}^{\bullet}, \Gamma_{L V}^{\bullet}, \Gamma_{V L}^{\bullet}, \Gamma_{V L V}^{\bullet}$ are stable and $\Gamma_{L V}^{\bullet} \subset \Gamma_{V L}^{\bullet}=: \bar{\Gamma}^{\bullet}, \bar{\Gamma}_{L}^{\bullet}=\bar{\Gamma}_{V}^{\bullet}=\bar{\Gamma}^{\bullet}$ and $\bar{\varphi}_{L}^{\bullet}=\bar{\varphi}_{V}^{\bullet}=\bar{\varphi}^{\bullet}$, where $\bar{\varphi}^{\bullet}:=\varphi_{V L}^{\bullet}$ (see 5.8). Moreover, one has (60) and if $\Gamma_{V}$ is stable; (61) and (62). For this reason one may consider $\bar{\Gamma}^{\bullet}$ and $\bar{\varphi}^{\bullet}$ instead of $\Gamma_{L V}$ and $\varphi_{L V}$, instead of $\Gamma_{L V}^{\bullet}$ and $\varphi_{L V}^{\bullet}$ or instead of $\Gamma_{V L V}$ and $\varphi_{V L V}$, indeed $\bar{\Gamma}^{\bullet}$ contains all of the other extensions and $\bar{\varphi} \bullet$ agrees with all integrals.

Theorem 6.4 [8, Chapter 4,Theorem 1.19] Let E be an Archimedean directed partially ordered vector space. Then E can be embedded in a Dedekind complete Riesz. space $\hat{E}$ :

There exists an injective linear $\gamma: E \rightarrow \hat{E}$ for which

(a) $a \geq 0 \Longleftrightarrow \gamma(a) \geq 0$,

(b) $\gamma(E)$ is orderdense in $\hat{E}$ (for the definition of order dense see the seventh footnote).

Consequently, suprema in $\gamma(E)$ are preserved in $\hat{E}$.

\section{Integration for functions with values in $\mathbb{R}$}

In this section $(X, \mathcal{A}, \boldsymbol{\mu})$ is a complete $\sigma$-finite measure space and $\mathbf{E}=\mathbf{F}=\mathbb{R}$.

We write $S$ for the vector space of simple functions from $X$ to $\mathbb{R}$ (see 4.33). Since $\mathbb{R}$ is a Banach lattice with $\sigma$-order continuous norm, $S_{V}$ is stable and $S_{L V}=S_{V L}$, $\varphi_{L V}=\varphi_{V L}$ (by Theorem 5.12). We write $\bar{S}=S_{V L}$ and $\bar{\varphi}=\varphi_{V L}$.

Theorem 7.1 $\bar{S}=\mathcal{L}^{1}(\mu)$ and $\bar{\varphi}(f)=\int f \mathrm{~d} \mu$ for all $f \in \bar{S}$.

Proof We prove that $S_{V L}^{+} \subset \mathcal{L}^{1}(\mu)^{+} \subset S_{L V}^{+}$and that $\varphi_{L V}(f)=\int f \mathrm{~d} \mu$ for all $f \in \mathcal{L}^{+}(\mu)$.

$S_{V}$ consists of the bounded integrable functions $f$ for which $\{x \in X: f(x) \neq 0\}$ has finite measure. By monotone convergence, we have $f \in \mathcal{L}^{1}(\mu)$ for every $f \in S_{V L}^{+}$.

Conversely, let $f \in \mathcal{L}^{1}(\mu)^{+}$; we prove $f \in S_{L V}^{+}$and $\varphi_{L V}(f)=\int f \mathrm{~d} \mu$. Let $t \in(1, \infty)$. For $n \in \mathbb{Z}$, put $A_{n}=\left\{x \in X: t^{n} \leq f(x)<t^{n+1}\right\}$. Then $\left(A_{n}\right)_{n \in \mathbb{Z}}$ forms a partition. Define $g:=\sum_{n \in \mathbb{Z}} t^{n} \mathbb{1}_{A_{n}}$ and $h:=t g$; then $g \leq f \leq h$. Since

$$
\sum_{n \in \mathbb{Z}} t^{n} \mu\left(A_{n}\right) \leq \sum_{n \in \mathbb{Z}} \int f \mathbb{1}_{A_{n}} \mathrm{~d} \mu=\int f \mathrm{~d} \mu,
$$


we have $g \in S_{L}$ and $\varphi_{L}(g) \leq \int f \mathrm{~d} \mu$. Also, $h=t g \in S_{L}$, and $\varphi_{L}(h)-\varphi_{L}(g)=$ $(t-1) \varphi_{L}(g) \leq(t-1) \int f \mathrm{~d} \mu$. By this and Lemma 3.7 it follows that $f \in S_{L V}$ and $\varphi_{L V}(f)=\int f \mathrm{~d} \mu$.

\section{Extensions of integrals on simple functions}

In this section $\mathbf{E}$ is a directed partially ordered vector space, $(\mathrm{X}, \mathcal{A}, \mu)$ is a complete $\sigma$-finite measure space and $\mathcal{I}, \mathbf{S}, \boldsymbol{\varphi}$ are as in $4.33(\mathbf{F}=\mathbf{E})$

In 8.1-8.8 for $f$ in $S_{L V}$ or $S_{V L}$ we discuss the relation between $f$ being almost everywhere equal to zero and $f$ having integral zero (i.e., either $\varphi_{L V}(f)=0$ or $\left.\varphi_{V L}(f)=0\right)$.

In 8.9 we show that under some conditions a function in $S_{V}$ multiplied with an integrable function with values in $\mathbb{R}$ is a function in $S_{L V}$.

In 8.11-8.13 we investigate the relation between the " $L V$ "-extension on simple functions with respect to $\mu$ and $v$, where $v=h \mu$ for some measurable $h: X \rightarrow$ $[0, \infty)$.

In 8.14 we discuss the relation between the " $L V$ "-extension simple functions with values in $E$ or in another partially ordered vector space $F$, when one makes the composition of a function in the extension with a $\sigma$-order continuous linear map $E \rightarrow F$.

In 8.15-8.17 we will prove that under certain conditions on $X$ the function $x \mapsto$ $F(x, \cdot)$ is in $S_{V}$ for all $F \in C(X \times T)$ and we relate that to convolution of certain finite measures with continuous functions on a topological group.

Theorem 8.1 Let $f: X \rightarrow E$ and $f=0$ a.e. If $f \in S_{L V}$, then $\varphi_{L V}(f)=0$. If $S_{V}$ is stable and $f \in S_{V L V}$, then $\varphi_{V L V}(f)=0$.

Proof Let $B=\{x \in X: f(x) \neq 0\}$. Then $B \in \mathcal{A}$ and $\mu(B)=0$.

(I) Assume $f \in S_{V}$. Choose $\sigma, \tau \in S$ with $\sigma \leq f \leq \tau$. Then $\sigma \mathbb{1}_{B}, \tau \mathbb{1}_{B} \in S$, $\sigma \mathbb{1}_{B} \leq f \leq \tau \mathbb{1}_{B}$, and $\varphi\left(\sigma \mathbb{1}_{B}\right)=\varphi\left(\tau \mathbb{1}_{B}\right)=0$. Hence $\varphi_{V}(f)=0$.

(II) Suppose $\sigma \in S_{L}^{+}$and $\left(A_{n}\right)_{n \in \mathbb{N}}$ is a $\varphi$-partition for $\sigma$. Then $\sigma \mathbb{1}_{A_{n} \cap B} \in S^{+}$for all $n \in \mathbb{N}$ and $\sum_{n} \varphi\left(\sigma \mathbb{1}_{A_{n} \cap B}\right)=0$, i.e., $\sigma \mathbb{1}_{B} \in S_{L}^{+}$with $\varphi_{L}\left(\sigma \mathbb{1}_{B}\right)=0$. In particular, if $f \in S_{L}$ then $\varphi_{L}(f)=0$.

(III) Assume $f \in S_{L V}$. With (II) one can repeat the argument of (I) with $S$ replaced by $S_{L}$ and conclude $\varphi_{L V}(f)=0$.

(IV) Suppose $S_{V}$ is stable and $f \in S_{V L V}$. One can repeat the argument in (III) with $S$ replaced by $S_{V}$ and conclude $\varphi_{V L V}(f)=0$.

Definition 8.2 A subset $D \subset E$ is called order bounded if there are $a, b \in E$ for which $a \leq D \leq b$.

Theorem 8.3 Let $f \in S_{L V}$ or (assuming $S_{V}$ is stable) $f \in S_{V L V}$. Then there exists a partition $\left(A_{n}\right)_{n \in \mathbb{N}}$ such that each set $f\left(A_{n}\right)$ is order bounded.

Proof There exists a partition $\left(A_{n}\right)_{n \in \mathbb{N}}$ such that for all $n \in \mathbb{N}$ there exist $h_{n}, g_{n} \in S$ for which $h_{n} \leq f \mathbb{1}_{A_{n}} \leq g_{n}$. Choose $a_{n}, b_{n} \in E$ for which $a_{n} \leq h_{n}(x)$ and $g_{n}(x) \leq b_{n}$ for all $x \in X$. Then $a_{n} \leq f(x) \leq b_{n}$ for $n \in \mathbb{N}, x \in A_{n}$. 
Theorem 8.4 Let $f: X \rightarrow E$ and $f=0$ a.e. Suppose there exists a partition $\left(A_{n}\right)_{n \in \mathbb{N}}$ such that for every $n \in \mathbb{N}$ the subset $f\left(A_{n}\right)$ of $E$ is order bounded. Then $f \in S_{L V}$ and if $S_{V}$ is stable then also $f \in \Gamma_{V L}$.

Proof Choose $a_{1}, a_{2}, \ldots$ and $b_{1}, b_{2}, \ldots$ in $E$ such that

$$
a_{n} \leq f(x) \leq b_{n} \quad\left(n \in \mathbb{N}, x \in A_{n}\right) .
$$

Let $B=\{x \in X: f(x) \neq 0\}$. Then $B \in \mathcal{A}$ and $\mu(B)=0$. Hence $g:=$ $\sum_{n \in \mathbb{N}} a_{n} \mathbb{1}_{A_{n} \cap B}$ and $h:=\sum_{n \in \mathbb{N}} b_{n} \mathbb{1}_{A_{n} \cap B}$ are elements of $S_{L}$ with $\varphi(g)=0$ and $\varphi_{L}(h)=0$. As $g \leq f \leq h$, we get $f \in S_{L V}$ and if $S_{V}$ is stable also $f \in S_{V L}$.

For a real valued function $f: X \rightarrow \mathbb{R}$ with $f \geq 0$ and $\int f \mathrm{~d} \mu=0$ we have $f=0$ a.e. We will give an example of a $f \in S_{V}^{+}$with $\varphi_{V}(f)=0$ but which is nowhere zero (Example 8.8). On the positive side, in Theorem 8.7 we show that $f=0$ a.e. if $f \in S_{L V}^{+}$and $\varphi_{L V}(f)=0$ provided that $E$ satisfies a certain separability condition.

Definition 8.5 We call a subset $D$ of $E^{+} \backslash\{0\}$ pervasive ${ }^{8}$ in $E$ if for all $a \in E$ with $a>0$ there exists a $d \in D$ such that $0<d \leq a$. We say that $E$ possesses a pervasive subset if there exists a pervasive $D \subset E^{+} \backslash\{0\}$.

Example 8.6 The Riesz spaces $\mathbb{R}^{\mathbb{N}}, \ell^{\infty}, c, c_{0}, \ell^{1}$ and $c_{00}$ possess countable pervasive subsets. Indeed, in each of them the set $\left\{\lambda e_{n}: \lambda \in \mathbb{Q}^{+}, \lambda>0, n \in \mathbb{N}\right\}$ is pervasive.

If $\mathcal{X}$ is a completely regular topological space, then $C(\mathcal{X})$ has a countable pervasive subset if and only if $\mathcal{X}$ has a countable base. (If $D \subset E^{+} \backslash\{0\}$ is countable and pervasive, then $\mathfrak{U}=\left\{f^{-1}(0, \infty): f \in D\right\}$ is a countable base; vise versa if $\mathfrak{U}$ is a countable base then with choosing an $f_{U}$ in $C(X)^{+}$for each $U \in \mathfrak{U}$ with $f_{U}=0$ on $U^{c}$ and $f_{U}(x)=1$ for some $x \in U$, the set $D=\left\{\varepsilon f_{U}: \varepsilon \in \mathbb{Q}, \varepsilon>0, U \in \mathfrak{U}\right\}$ is pervasive.)

$L^{1}(\lambda)$ and $L^{\infty}(\lambda)$ do not possess countable pervasive subsets, considering the Lebesgue measure space $(\mathbb{R}, \mathcal{M}, \lambda)$. (Suppose one of them does. Then one can prove the existence of non-negligible measurable sets $A_{1}, A_{2}, \ldots \in \mathcal{M}$ such that every non-negligible measurable set contains an $A_{n}$, whereas $\lambda\left(A_{n}\right)<2^{-n}$ for all $n \in \mathbb{N}$. Putting $C=\mathbb{R} \backslash \bigcup_{n \in \mathbb{N}} A_{n}$ we have a non-negligible measurable set that contains no $A_{n}$ : a contradiction.)

Theorem 8.7 Let $E$ possess a countable pervasive subset D. Let $f \in S_{L V}$. Let $\Lambda, \Upsilon \subset S_{L}$ be countable sets such that $\Lambda \leq f \leq \Upsilon$ and $\sup \varphi_{L}(\Lambda)=\inf \varphi_{L}(\Upsilon)$. Then for almost all $x \in X$

$$
\sup _{g \in \Lambda} g(x)=f(x)=\inf _{h \in \Upsilon} h(x) .
$$

Consequently, if $f \in S_{L V}^{+}$and $\varphi_{L V}(f)=0$, then $f=0$ a.e. (However, see Example 8.8.)

8 Our use of the term is similar to the one of van Gaans and Kalauch in [12, Definition 2.3]. 
Proof (I) First, as a special case (namely $f=0$ ), let $\left(\tau_{n}\right)_{n \in \mathbb{N}}$ be a sequence in $S_{L}$ with $\tau_{n} \geq 0$ for all $n \in \mathbb{N}$ and $\inf _{n \in \mathbb{N}} \varphi_{L}\left(\tau_{n}\right)=0$. We prove that $\inf _{n \in \mathbb{N}} \tau_{n}(x)=0$ for almost all $x \in X$, by proving that $\mu(A)=0$, where $A$ is the complement of the set $\left\{x \in X: \inf _{n \in \mathbb{N}} \tau_{n}(x)=0\right\}$. Indeed, for this $A$ we have

$$
A=\bigcup_{d \in D} A_{d}, \quad \text { with } \quad A_{d}=\bigcap_{n \in N}\left\{x \in X: d \leq \tau_{n}(x)\right\} .
$$

Note that for all $n \in \mathbb{N}$ and $d \in D$ the set $\left\{x \in X: d \leq \tau_{n}(x)\right\}$ is measurable. Furthermore, for all $d \in D$ we have:

$$
d \mu\left(A_{d}\right)=\varphi\left(d \mathbb{1}_{A_{d}}\right) \leq \varphi_{L}\left(\tau_{n}\right) \quad(n \in \mathbb{N})
$$

Hence $\mu\left(A_{d}\right)=0$ for all $d \in D$ and thus $\mu(A)=0$.

(II) Suppose that $\Lambda, \Upsilon \subset \Gamma_{L}$ are countable sets such that $\Lambda \leq f \leq \Upsilon$, $\sup \varphi_{L}(\Lambda)=$ $\inf \varphi_{L}(\Upsilon)$. Then $\inf \varphi_{L}(\Upsilon-\Lambda)=0$, so by (I) $\inf _{g \in \Upsilon, h \in \Lambda}(g(x)-h(x))=0$ for almost all $x \in X$.

Example 8.8 We give an example of a $f \in S_{V}^{+}$with $\varphi_{V}(f)=0$, where $f \neq 0$ everywhere. Let $([0,1), \mathcal{M}, \lambda)$ be the Lebesgue measure space with underlying set $[0,1)$. Let $E=\ell^{\infty}([0,1))$ (see Sect. 2). Let $f: \mathbb{R} \rightarrow E^{+}$be defined by $f(t)=\mathbb{1}_{\{t\}}$ for $t \in[0,1)$. Note that $f$ is not partially in $S$. We will show $f \in S_{V}$. For $n \in \mathbb{N}$ make $\tau_{n} \in S:$

$$
\tau_{n}(t)=\mathbb{1}_{\left[\frac{i-1}{n}, \frac{i}{n}\right)} \quad \text { if } i \in\{1, \ldots, n\}, t \in\left[\frac{i-1}{n}, \frac{i}{n}\right)
$$

Then $\varphi\left(\tau_{n}\right)=\frac{1}{n} \mathbb{1}_{[0,1)}$ and $0 \leq f \leq \tau_{n}$ for $n \in \mathbb{N}$, so $f \in S_{V}$ and $\varphi_{V}(f)=0$. But $f(t) \neq 0$ for all $t$.

Theorem 8.9 Let $E$ be Archimedean and mediated. Let $f: X \rightarrow E$ and $g: X \rightarrow \mathbb{R}$. We write $g f$ for the function $x \mapsto g(x) f(x)$. Then

(a) $f \in S_{V}$ and $g$ is bounded and measurable $\Longrightarrow g f \in S_{V}$.

(b) $f$ is partially in $S_{V}$ and $g$ is measurable $\Longrightarrow g f$ is partially in $S_{V}$.

(c) $f \in S_{V}$ and $g \in \mathcal{L}^{1}(\mu) \Longrightarrow g f \in S_{L V}$.

(d) $f \in S_{V L}$ and $g$ is bounded and measurable $\Longrightarrow g f \in S_{V L}$.

(e) $f \in S_{V L}, f(X)$ is order bounded and $g \in \mathcal{L}^{1}(\mu) \Longrightarrow g f \in S_{V L}$.

Proof $E$ is splitting (see 4.23(b)).

(a) is a consequence of Theorem 3.21(a) (see also Remark 3.22).

(b) Let $\left(A_{n}\right)_{n \in \mathbb{N}}$ be a partition such that $f \mathbb{1}_{A_{n}} \in S_{V}$ and $g \mathbb{1}_{A_{n}}$ is bounded for all $n \in \mathbb{N}$. By (a) every $g f \mathbb{1}_{A_{n}}$ lies in $S_{V}$. Then $g f$ is partially in $S_{V}$.

(c) Assume $f \geq 0$ and $g \geq 0$. Choose (see the proof of Theorem 7.1) a partition $\left(A_{n}\right)_{n \in \mathbb{N}}$ and numbers $\lambda_{1}, \lambda_{2}, \ldots$ in $[0, \infty)$ with

$$
\tau:=\sum_{n \in \mathbb{N}} \lambda_{n} \mathbb{1}_{A_{n}} \geq g, \quad \sum_{n \in \mathbb{N}} \lambda_{n} \mu\left(A_{n}\right)<\infty .
$$


Then $\tau s \in S_{L}$ for all $s \in S$. Choose $s \in S$ with $s \geq f$. Then $0 \leq g f \leq \tau s$. From Theorem 5.5(e) and (b) it follows that $g f \in S_{L V}$.

(d) Assume $f \geq 0$ and $0 \leq g \leq \mathbb{1}$. Using (b), choose a partition $\left(A_{n}\right)_{n \in \mathbb{N}}$ with $f \mathbb{1}_{A_{n}} \in S_{V}$ and $g f \mathbb{1}_{A_{n}} \in S_{V}$ for all $n \in \mathbb{N}$. Then

$$
0 \leq \varphi_{V}\left(g f \mathbb{1}_{A_{n}}\right) \leq \varphi_{V}\left(f \mathbb{1}_{A_{n}}\right) \quad(n \in \mathbb{N}) .
$$

Since $\sum_{n} \varphi_{V}\left(f \mathbb{1}_{A_{n}}\right)$ exists and $E$ is splitting, $\sum_{n} \varphi_{V}\left(g f \mathbb{1}_{A_{n}}\right)$ exists.

(e) Assume $f \geq 0$ and $g \geq 0$. Choose $a \in E^{+}$with $f(x) \leq a$ for all $x \in X$. Choose a partition $\left(A_{n}\right)_{n \in \mathbb{N}}$ and $\lambda_{1}, \lambda_{2}, \ldots \in[0, \infty)$ with

$$
\begin{aligned}
& g f \mathbb{1}_{A_{n}} \in S_{V} \quad(n \in \mathbb{N}), \\
& g \leq \sum_{n \in \mathbb{N}} \lambda \mathbb{1}_{A_{n}}, \quad \sum_{n \in \mathbb{N}} \lambda_{n} \mu\left(A_{n}\right)<\infty \quad \text { (see the proof of Theorem 7.1). }
\end{aligned}
$$

Then

$$
\begin{aligned}
& g f \mathbb{1}_{A_{n}} \leq \lambda_{n} a \mathbb{1}_{A_{n}} \quad(n \in \mathbb{N}), \\
& \varphi_{V}\left(\lambda_{n} a \mathbb{1}_{A_{n}}\right)=\varphi\left(\lambda_{n} a \mathbb{1}_{A_{n}}\right)=\lambda_{n} \mu\left(A_{n}\right) a \quad(n \in \mathbb{N}),
\end{aligned}
$$

so $\sum_{n} \varphi_{V}\left(\lambda_{n} a \mathbb{1}_{A_{n}}\right)$ exists and so does $\sum_{n} \varphi_{V}\left(g f \mathbb{1}_{A_{n}}\right)$.

8.10 In Lemma 8.11, Theorems 8.12 and 8.13 we investigate the relation between the extensions $S_{L V}$ generated by two different measures, namely $\mu$ and $h \mu$ for a measurable function $h: X \rightarrow[0, \infty)$.

Note that for such a function $h$ and all $s \in(1, \infty)$ there exists a $j: X \rightarrow[0, \infty)$ that is partially in the space of simple functions $X \rightarrow[0, \infty)$, i.e., $j=\sum_{n \in \mathbb{N}} \alpha_{n} \mathbb{1}_{A_{n}}$ for a partition $\left(A_{n}\right)_{n \in \mathbb{N}}$ and $\left(\alpha_{n}\right)_{n \in \mathbb{N}}$ in $[0, \infty)$ (or in the language of $3.16 j$ is partially in $[\mathcal{A}]$ ) for which $j \leq h \leq s j$. In the following $(8.11,8.12$ and 8.13) we will write $\mathcal{I}^{\mu}, S^{\mu}$ and $\varphi^{\mu}$ instead of $\mathcal{I}, S$ and $\varphi$ and, similarly for another measure $\nu$ on $(X, \mathcal{A})$, we write $\mathcal{I}^{\nu}, S^{\nu}$ and $\varphi^{\nu}$ according to 4.33 with $\nu$ instead of $\mu$.

Lemma 8.11 Suppose $E$ is splitting. Let $h: X \rightarrow[0, \infty)$ be measurable, $v:=h \mu$. Let $s \in(1, \infty)$ and let $j: X \rightarrow[0, \infty)$ be partially in $[\mathcal{A}]$ and such that $j \leq h \leq s j$. Let $f \in S_{L}^{\nu+}$. Then $j f \in S_{L}^{\mu}$ and $\varphi_{L}^{\mu}(j f) \leq \varphi_{L}^{\nu}(f) \leq s \varphi_{L}^{\mu}(j f)$.

Proof Assume $\left(A_{n}\right)_{n \in \mathbb{N}}$ is a partition for $j$ and a $\varphi^{\mu}$-partition for $f$ (so $\left(A_{n}\right)_{n \in \mathbb{N}}$ is in $\mathcal{I}^{v} \cap \mathcal{I}^{\mu}$, i.e., $\mu\left(A_{n}\right), v\left(A_{n}\right)<\infty$ for all $\left.n \in \mathbb{N}\right)$. Choose $\left(\alpha_{n}\right)_{n \in \mathbb{N}}$ in $[0, \infty)$ and $\left(b_{n}\right)_{n \in \mathbb{N}}$ in $E^{+}$such that

$$
j=\sum_{n \in \mathbb{N}} \alpha_{n} \mathbb{1}_{A_{n}}, \quad f=\sum_{n \in \mathbb{N}} b_{n} \mathbb{1}_{A_{n}} .
$$


Then $j f=\sum_{n \in \mathbb{N}} \alpha_{n} b_{n} \mathbb{1}_{A_{n}}$ and thus is in $S_{L}^{\mu}$ if $\sum_{n} \mu\left(A_{n}\right) \alpha_{n} \beta_{n}$ exists in $E$. For each $n \in \mathbb{N}$

$$
0 \leq \mu\left(A_{n}\right) \alpha_{n}=\int j \mathbb{1}_{A_{n}} \mathrm{~d} \mu \leq \int h \mathbb{1}_{A_{n}} \mathrm{~d} \mu=v\left(A_{n}\right)
$$

whence $0 \leq \mu\left(A_{n}\right) \alpha_{n} b_{n} \leq v\left(A_{n}\right) b_{n}$. Because $f \in S_{L}^{\nu+}, \sum_{n} v\left(A_{n}\right) b_{n}$ exists in $E$. Since $E$ is splitting also $\sum_{n} \mu\left(A_{n}\right) \alpha_{n} b_{n}$ exists in $E$, i.e., $j f \in S_{L}^{\mu}$.

Furthermore, $\varphi_{L}^{\mu}(j f)=\sum_{n} \mu\left(A_{n}\right) \alpha_{n} b_{n} \leq \sum_{n} v\left(A_{n}\right) b_{n}=\varphi_{L}^{v}(f)$. On the other hand, we get $\mu\left(A_{n}\right) \alpha_{n}=\int j \mathbb{1}_{A_{n}} \mathrm{~d} \mu \geq \frac{1}{s} \int h \mathbb{1}_{A_{n}} \mathrm{~d} \mu=\frac{1}{s} \nu\left(A_{n}\right)$ for each $n \in \mathbb{N}$ : it follows that $\varphi_{L}^{\mu}(j f) \geq \frac{1}{s} \varphi_{L}^{\nu}(f)$.

Theorem 8.12 Let $E$ be Archimedean and splitting. Let $h: X \rightarrow[0, \infty)$ be measurable, $v:=h \mu$.

(a) $f \in S_{L V}^{v} \Longrightarrow h f \in S_{L V}^{\mu}, \varphi_{L V}^{\mu}(h f)=\varphi_{L V}^{v}(f)$,

(b) $f \in S_{V L}^{v} \Longrightarrow h f \in S_{V L}^{\mu}, \varphi_{V L}^{\mu}(h f)=\varphi_{V L}^{v}(f)$.

Proof Since both $S_{L V}^{\nu}$ and $S_{V L}^{\nu}$ are directed, we assume $f \geq 0$.

(a) Let $f \in S_{L V}^{\nu+}$. For $n \in \mathbb{N}$ let $j_{n}$ be partially in $[\mathcal{A}]$ and such that $j_{n} \leq h \leq\left(1+\frac{1}{n}\right) j_{n}$. Let $\Lambda, \Upsilon \subset S_{L}^{v}$ be countable sets with $\Lambda \leq f \leq \Upsilon$ be such that $\sup \varphi_{L}^{v}(\Lambda)=$ $\varphi_{L V}^{\nu}(f)=\inf \varphi_{L}^{\nu}(\Upsilon)$. Then for all $\sigma \in \Lambda$ (note that $\left.\sigma \in S_{L}^{\nu+}-S_{L}^{\nu+}\right), \tau \in \Upsilon$ and $n \in \mathbb{N}$ we have $j_{n} \sigma \leq h f \leq\left(1+\frac{1}{n}\right) j_{n} \tau$ and by Lemma $8.11 j_{n} \sigma$ and $\left(1+\frac{1}{n}\right) j_{n} \tau$ are in $S_{L}^{\mu}$. Therefore we are done if both $\inf _{n \in \mathbb{N}, \sigma \in \Lambda, \tau \in \Upsilon} \varphi_{L}^{\mu}\left(\left(1+\frac{1}{n}\right) j_{n} \tau-j_{n} \sigma\right)=0$ and $\varphi_{L}^{\mu}\left(j_{n} \sigma\right) \leq \varphi_{L V}^{\nu}(f) \leq \varphi_{L}^{\mu}\left(\left(1+\frac{1}{n}\right) j_{n} \tau\right)$ for all $n \in \mathbb{N}$ and all $\sigma \in \Lambda, \tau \in \Upsilon$. By Lemma 8.11 applied repeatedly we have

$$
\begin{aligned}
0 & \leq \varphi_{L}^{\mu}\left(\left(1+\frac{1}{n}\right) j_{n} \tau-j_{n} \sigma\right)=\varphi_{L}^{\mu}\left(j_{n} \tau-j_{n} \sigma\right)+\frac{1}{n} \varphi_{L}^{\mu}\left(j_{n} \tau\right) \\
& \leq \varphi_{L}^{v}(\tau-\sigma)+\frac{1}{n} \varphi_{L}^{\nu}(\tau)
\end{aligned}
$$

which has infimum 0 since $E$ is Archimedean and $\inf _{\tau \in \Upsilon, \sigma \in \Lambda} \varphi_{L}^{v}(\tau-\sigma)=0$. On the other hand, by Lemma 8.11,

$$
\begin{gathered}
\varphi^{\mu}\left(j_{n} \sigma\right) \leq \varphi_{L}^{\nu}(\sigma) \leq \varphi_{L V}^{\nu}(f) \leq \varphi_{L}^{\nu}(\tau) \leq\left(1+\frac{1}{n}\right) \varphi_{L}^{\mu}\left(j_{n} \tau\right) \\
(n \in \mathbb{N}, \sigma \in \Lambda, \tau \in \Upsilon) .
\end{gathered}
$$

(b) Let $f \in S_{V L}^{\nu+}$. Choose a partition $\left(A_{n}\right)_{n \in \mathbb{N}}$ with $f \mathbb{1}_{A_{n}} \in S_{V}^{\nu}$ for $n \in \mathbb{N}$. By (a), $h f \mathbb{1}_{A_{n}} \in S_{L V}^{\mu}$ for $n \in \mathbb{N}$; by Lemma $5.7 h f \mathbb{1}_{A_{n}}$ is partially in $S_{V}^{\mu}$.

Therefore we can choose a partition $\left(B_{n}\right)_{n \in \mathbb{N}}$ with

$$
f \mathbb{1}_{B_{n}} \in S_{V}^{\nu}, \quad h f \mathbb{1}_{B_{n}} \in S_{V}^{\mu} \quad(n \in \mathbb{N}) .
$$

By (a), $\varphi_{V}^{\nu}\left(f \mathbb{1}_{B_{n}}\right)=\varphi_{V}^{\mu}\left(h f \mathbb{1}_{B_{n}}\right)$ for all $n \in \mathbb{N}$. But $f \in S_{V L}^{\nu+}$, so

$$
\varphi_{V L}^{v}(f)=\sum_{n} \varphi_{V}^{v}\left(f \mathbb{1}_{B_{n}}\right)=\sum_{n} \varphi_{V}^{\mu}\left(h f \mathbb{1}_{B_{n}}\right) .
$$

Then $h f \in S_{V L}^{\mu}$ and $\varphi_{V L}^{\mu}(h f)=\varphi_{V L}^{v}(f)$. 
Theorem 8.13 Let $E$ be Archimedean and splitting. Let $h: X \rightarrow[0, \infty)$ be measurable, $v:=h \mu, A=\{x \in X: h(x)>0\}$. Let $f: X \rightarrow E$ be such that $h f \in S_{L V}^{\mu}$. Then $f \mathbb{1}_{A} \in S_{L V}^{v}$.

Proof Define $h^{*}: X \rightarrow[0, \infty)$ by

$$
h^{*}(x)= \begin{cases}\frac{1}{h(x)} & \text { if } x \in A, \\ 0 & \text { if } x \notin A .\end{cases}
$$

Then $h^{*}$ is measurable and $h h^{*}=\mathbb{1}_{A}$ and $\mathbb{1}_{A}=\mathbb{1} v$-a.e.

$h f$ is in $S_{L}^{\mu}$ and thus in $S_{L}^{\mathbb{1}_{A} \mu}$, and since $\mathbb{1}_{A} \mu=h^{*} \nu$, also $h f \in S_{L}^{h^{*} \nu}$. By Theorem 8.12, applied to $h^{*}, h^{*} v, v, h f$ instead of $h, v, \mu, f$, the function $h^{*} h f$ is an element of $S_{L V}^{v}$. But $h^{*} h f=\mathbb{1}_{A} f$.

In Theorem 8.14 we show that extensions of simple functions with values in $E$ composed with a $\sigma$-order continuous linear map $E \rightarrow F$ are extensions of simple functions with values in $F$ (where $E$ and $F$ are Riesz spaces).

Theorem 8.14 Let $E$ and $F$ be Riesz spaces. Let $S^{E}$ and $\varphi^{E}$ be as in 4.33, and let $S^{F}$ and $\varphi^{F}$ be defined analogously. Let $\mathcal{L}_{c}(E, F)$ denote the set of $\sigma$-order continuous linear functions $E \rightarrow F$ and $E_{c}^{\sim}=\mathcal{L}_{c}(E, \mathbb{R})$ (definition and notation as in Zaanen [13, Chapter 12,§84]). Let $f \in S_{L V}^{E}$. Then $\alpha \circ f \in S_{L V}^{F}$ for all $\alpha \in \mathcal{L}_{c}(E, F)$ and

$$
\alpha\left(\varphi_{L V}^{E}(f)\right)=\varphi_{L V}^{F}(\alpha \circ f)
$$

In particular, $\alpha \circ f$ is integrable for all $\alpha \in E_{c}^{\sim}$, and $\alpha\left(\varphi_{L V}^{E}(f)\right)=\int \alpha \circ f \mathrm{~d} \mu$.

Proof Suppose $\alpha \in \mathcal{L}_{c}(E, F)^{+}$. Let $\tau \in S_{L}^{E+}$. Suppose $\tau=\sum_{n \in \mathbb{N}} a_{n} \mathbb{1}_{A_{n}}$ for some partition $\left(A_{n}\right)_{n \in \mathbb{N}}$ and a sequence $\left(a_{n}\right)_{n \in \mathbb{N}}$ in $E^{+}$. Then $\alpha\left(\varphi_{L}^{E}(\tau)\right)=$ $\alpha\left(\sum_{n} \mu\left(A_{n}\right) a_{n}\right)=\sum_{n} \mu\left(A_{n}\right) \alpha\left(a_{n}\right)$. Thus $\alpha \circ \tau$ is in $S_{L V}^{F}$ with $\alpha\left(\varphi_{L}^{E}(\tau)\right)=\varphi_{L}^{F}(\alpha \circ \tau)$. Let $\left(\sigma_{n}\right)_{n \in \mathbb{N}},\left(\tau_{n}\right)_{n \in \mathbb{N}}$ be sequences in $S_{L}^{E}$ with $\sigma_{n} \leq f \leq \tau_{n}, \sigma_{n} \uparrow, \tau_{n} \downarrow$ and $\varphi_{L V}^{E}(f)=$ $\sup _{n \in \mathbb{N}} \varphi_{L}^{E}\left(\sigma_{n}\right)=\inf _{n \in \mathbb{N}} \varphi_{L}^{E}\left(\tau_{n}\right)$. Then we have $\alpha\left(\varphi_{L V}^{E}(f)\right)=\sup _{n \in \mathbb{N}} \alpha\left(\varphi_{L}^{E}\left(\sigma_{n}\right)\right)=$ $\sup _{n \in \mathbb{N}} \varphi_{L}^{F}\left(\alpha \circ \sigma_{n}\right)$ and $\alpha\left(\varphi_{L V}^{E}(f)\right)=\inf _{n \in \mathbb{N}} \alpha\left(\varphi_{L}^{E}\left(\tau_{n}\right)\right)=\inf _{n \in \mathbb{N}} \varphi_{L}^{F}\left(\alpha \circ \tau_{n}\right)$. Since $\alpha \circ \sigma_{n} \leq \alpha \circ f \leq \alpha \circ \tau_{n}$ for all $n \in \mathbb{N}$, we conclude that $\alpha \circ f \in\left(S^{F}\right)_{L V}$ (see Theorem 7.1) with $\alpha\left(\varphi_{L V}^{E}(f)\right)=\varphi_{L V}^{F}(f)$.

Theorem 8.14 will be used in Sect. 9 to compare the integrals $\varphi_{L V}$ and $\varphi_{V L}$ with the Pettis integral.

Before proving Theorem 8.16 we state (in Theorem 8.15 ) that there is an equivalent formulation for a function $F$ to be in $C(X \times T)$ whenever $X, T$ are topological spaces and $X$ is compact.

Theorem 8.15 ([14, Theorem 7.7.5]) Let $X$ be a compact and let $T$ be a topological space. Let $F: X \times T \rightarrow \mathbb{R}$ be such that $F(\cdot, t) \in C(X)$ for all $t \in T$. Then $F \in C(X \times T)$ if and only if $t \mapsto F(\cdot, t)$ is continuous, where $C(X)$ is equipped with the supremum norm. Consequently, if $A \subset X$ is a compact set, then $t \mapsto \sup F(A, t)$ and $t \mapsto \inf F(A, t)$ are continuous. 
Theorem 8.16 Let $(X, d, \mu)$ be a compact metric probability space. Let $T$ be a topological space and $F \in C(X \times T)$. The function $H: X \rightarrow C(T)$ given by $H(x)=F(x, \cdot)$ is an element of $S_{V}$. Furthermore, for $t \in T, x \mapsto F(x, t)$ is integrable and

$$
\left[\varphi_{V}(H)\right](t)=\int F(x, t) \mathrm{d} \mu(x) \quad(t \in T) .
$$

Proof For $k \in \mathbb{N}$ let $A_{k 1}, \ldots, A_{k n_{k}}$ be a partition of $X$ with diam $A_{k i} \leq k^{-1}$. Define

$$
\Delta_{k}(t)=\sup _{x, y \in X, d(x, y)<k^{-1}}|F(x, t)-F(y, t)| \quad(t \in T) .
$$

Since $x \mapsto F(x, t)$ is uniformly continuous for all $t \in T, \Delta_{k}(t) \downarrow 0$ for all $t \in T$. By Theorem 8.15t $\mapsto \sup F\left(A_{k i}, t\right)$ and $t \mapsto \inf F\left(A_{k i}, t\right)$ are continuous for all $k \in \mathbb{N}$ and $i \in\left\{1, \ldots, n_{k}\right\}$. For $k \in \mathbb{N}$ let $h_{k}, l_{k}: X \rightarrow C(T)$ be given by

$$
\begin{aligned}
h_{k}(x) & =t \mapsto \sup F\left(A_{k i}, t\right) \quad\left(x \in A_{k i}\right), \\
l_{k}(x)=t & \mapsto \inf F\left(A_{k i}, t\right) \quad\left(x \in A_{k i}\right) .
\end{aligned}
$$

Then $h_{k}, l_{k} \in S$ and $\left(h_{k}(x)\right)(t) \geq F(x, t) \geq\left(l_{k}(x)\right)(t)$ for all $x \in X, t \in T$. For $x \in A_{k i} \cap A_{m j}$ and $t \in T$

$$
\begin{aligned}
\left(h_{k}(x)-l_{m}(x)\right)(t) & =\sup F\left(A_{k i}, t\right)-\inf F\left(A_{m j}, t\right) \\
& \leq \sup \left\{F(u, t)-F(v, t): u, v \in A_{k i} \cup A_{m j}\right\} \leq \Delta_{k \wedge m}(t) .
\end{aligned}
$$

Let $a_{k}=\varphi\left(h_{k}\right)$ and $b_{k}=\varphi\left(l_{k}\right)$ for $k \in \mathbb{N}$. Then $0 \leq a_{k}(t)-b_{m}(t) \leq \Delta_{k \wedge m}(t)$ for all $k, m \in \mathbb{N}$ and $\inf _{k, m \in \mathbb{N}} a_{k}(t)-b_{m}(t) \leq \inf _{k \in \mathbb{N}} \Delta_{k}(t)=0$. Since $a_{k}, b_{k} \in C(T)$ and $\sup _{n \in \mathbb{N}} b_{n}(t)=\inf _{n \in \mathbb{N}} a_{n}(t)$ for all $t \in T$, the function $t \mapsto \inf _{n \in \mathbb{N}} a_{n}(t)$ is continuous, i.e., $x \mapsto F(x, \cdot)$ is an element of $S_{V}$. Furthermore, we conclude that the function $x \mapsto F(x, t)$ is integrable (by Theorem 7.1) and conclude (83).

Example 8.17 Consider a metrisable locally compact group $G$. Let $X \subset G$ be a compact set and $\mu$ be a finite (positive) measure on $\mathcal{B}(X)$, the Borel- $\sigma$-algebra of $X$. Let $g \in C(G)$. Define the convolution of $g$ and $\mu$ to be the function $g * \mu: G \rightarrow \mathbb{R}$ given by $g * \mu(t)=\int g\left(t x^{-1}\right) \mathrm{d} \mu(x)$ for $t \in G$. For $x \in X$, let $L_{x} g \in C(G)$ be the function $t \mapsto g\left(t x^{-1}\right)$. Then by Theorem 8.16, the function $f: X \rightarrow C(G)$ given by $f(x)=L_{x} g$ is in $S_{V}$ and $g * \mu=\varphi_{V}(f) \in C(G)$.

\section{Comparison with Bochner- and Pettis integral}

We consider the situation of Sect. 8, with an $\mathbf{E}$ that has the structure of a Banach lattice. We write $\|\cdot\|$ for the norm on $E$ and $E^{\prime}$ for the dual of $E$. Then, next to our $\varphi_{L V}$ (and other extensions) there are the Bochner and the Pettis integrals. (We refer the reader to Hille and Phillips [3, Section 3.7] for background on both integrals.) 
We denote the set of Bochner (Pettis) integrable functions from the measure space $(X, \mathcal{A}, \mu)$ into the Banach lattice $E$ by $\mathfrak{B}(\mathfrak{P})$ and the Bochner (Pettis) integral of an integrable function $f$ by $\mathfrak{b}(f)(\mathfrak{p}(f))$.

9.1 By definition of the Bochner integral, where one also starts with defining the integral on simple functions: $S \subset \mathfrak{B}$ and $\varphi=\mathfrak{b}$ on $S$. Since $\mathfrak{B} \subset \mathfrak{P}$ and $\mathfrak{b}=\mathfrak{p}$ on $\mathfrak{B}$ we also have $S \subset \mathfrak{P}$ with $\varphi=\mathfrak{p}$ on $S$.

9.2 The following is used in this section. The Banach dual of $E$ is equal to the order dual, i.e., $E^{\prime}=E^{\sim}$. Moreover, for $x, y \in E$ (see de Jonge and van Rooij [15, Theorem 10.2])

$$
x \leq y \quad \Longleftrightarrow \quad \alpha(x) \leq \alpha(y) \text { for all } \alpha \in E^{\sim+}
$$

This implies that for a sequence $\left(y_{n}\right)_{n \in \mathbb{N}}$ and $x, y$ in $E$ :

$$
\inf _{n \in \mathbb{N}} \alpha\left(y_{n}\right)=0 \text { for all } \alpha \in E^{\sim+} \Longrightarrow \inf _{n \in \mathbb{N}} y_{n}=0 \text {. }
$$

Theorem 9.3 Let $f \in \mathfrak{P}^{+}$and $f$ be partially in $S$. Then $f \in S_{L}^{+}$and $\mathfrak{p}(f)=\varphi_{L}(f)$.

Proof Let $\left(A_{n}\right)_{n \in \mathbb{N}}$ be a partition for which $f_{n}:=f \mathbb{1}_{A_{n}} \in S$. Then for every $\alpha \in E^{\sim+}$

$$
\alpha(\mathfrak{p}(f))=\int \alpha \circ f \mathrm{~d} \mu=\sum_{n \in \mathbb{N}} \int \alpha \circ f_{n} \mathrm{~d} \mu=\sum_{n \in \mathbb{N}} \alpha\left(\varphi\left(f_{n}\right)\right) .
$$

Hence $\inf _{N \in \mathbb{N}} \alpha\left(\mathfrak{p}(f)-\sum_{n=1}^{N} \varphi\left(f_{n}\right)\right)=0$ and thus $\mathfrak{p}(f)=\sum_{n} \varphi\left(f_{n}\right)$ (see (88)).

Theorem 9.4 Let $f \in \mathfrak{P}$. Then the following holds.

(a) If $g \in S_{L V}$ and $f \leq g$, then $\mathfrak{p}(f) \leq \varphi_{L V}(g)$.

(b) If $S_{V}$ is stable, $g \in S_{V L V}$ and $f \leq g$, then $\mathfrak{p}(f) \leq \varphi_{V L V}(g)$.

Consequently, $\mathfrak{p}=\varphi_{L V}$ on $\mathfrak{P} \cap S_{L V}$, and $\mathfrak{p}=\varphi_{V L V}$ on $\mathfrak{P} \cap S_{V L V}$ if $S_{V}$ is stable.

The statements in (a) and (b) remain valid by replacing all " $\leq$ ” by " $\geq$ ".

Proof It will be clear that if $g \in S$ and $f \leq g$, then $g \in \mathfrak{P}$ and hence $\mathfrak{p}(f) \leq \mathfrak{p}(g)=$ $\varphi(g)$.

If $g \in S_{V}$ and $f \leq g$, then there exists an $\Upsilon \subset S$ with $g \leq \Upsilon$ and $\varphi_{V}(g)=$ $\inf \varphi(\Upsilon)=\inf \mathfrak{p}(\Upsilon) \geq \mathfrak{p}(f)$.

Let $g \in S_{L}$ and assume $f \leq g$. Let $g_{1}, g_{2} \in S_{L}^{+}$be such that $g=g_{1}-g_{2}$. Let $\left(B_{i}\right)_{i \in \mathbb{N}}$ be a $\varphi$-partition for both $g_{1}$ and $g_{2}$. Write $A_{n}=\bigcup_{i=1}^{n} B_{i}$ for $n \in \mathbb{N}$. Let $\alpha \in E^{\sim+} . \alpha \circ\left(f \mathbb{1}_{A}\right)=(\alpha \circ f) \mathbb{1}_{A}$ for every $A \in \mathcal{A}$, so that $\alpha \circ\left(f \mathbb{1}_{A}\right)$ is integrable. Thus, for $n \in \mathbb{N}$ we have 


$$
\begin{aligned}
\int(\alpha \circ f) \mathbb{1}_{A_{n}} \mathrm{~d} \mu & =\int \alpha \circ\left(f \mathbb{1}_{A_{n}}\right) \mathrm{d} \mu \leq \int \alpha \circ\left(g \mathbb{1}_{A_{n}}\right) \mathrm{d} \mu \\
& =\int \alpha \circ g_{1} \mathbb{1}_{A_{n}} \mathrm{~d} \mu-\int \alpha \circ g_{2} \mathbb{1}_{A_{n}} \mathrm{~d} \mu \\
& =\alpha\left(\varphi\left(g_{1} \mathbb{1}_{A_{n}}\right)\right)-\alpha\left(\varphi\left(g_{2} \mathbb{1}_{A_{n}}\right)\right) \\
& \leq \alpha\left(\varphi\left(g_{1} \mathbb{1}_{A_{m}}\right)\right)-\alpha\left(\varphi\left(g_{2} \mathbb{1}_{A_{k}}\right)\right) \quad(k, m \in \mathbb{N}, k<n<m) .
\end{aligned}
$$

Which implies that $\int(\alpha \circ f) \mathbb{1}_{A_{n}} \mathrm{~d} \mu+\alpha\left(\varphi\left(g_{2} \mathbb{1}_{A_{k}}\right)\right) \leq \alpha\left(\varphi_{L}\left(g_{1}\right)\right)$ as soon as $k<n$. By letting $n$ tend to $\infty$ (as $\left.\int(\alpha \circ f) \mathbb{1}_{A_{n}} \mathrm{~d} \mu \rightarrow \int \alpha \circ f \mathrm{~d} \mu=\alpha(\mathfrak{p}(f))\right)$, for each $k \in \mathbb{N}$ we obtain

$$
\alpha(\mathfrak{p}(f)) \leq \alpha\left(\varphi_{L}\left(g_{1}\right)-\varphi\left(g_{2} \mathbb{1}_{A_{k}}\right)\right) .
$$

This holds for all $\alpha \in E^{\sim+}$, so

$$
\mathfrak{p}(f) \leq \varphi_{L}\left(g_{1}\right)-\varphi\left(g_{2} \mathbb{1}_{A_{k}}\right)
$$

This, in term is true for every $k, \operatorname{so} \mathfrak{p}(f)) \leq \varphi_{L}(g)$.

We leave it to check that the preceding lines can be repeated with $S_{V}, S_{L}$ or $S_{V L}$ instead of $S$.

Theorem 9.5 Suppose $\|\cdot\|$ is $\sigma$-order continuous. Write $\bar{S}=S_{L V}=S_{V L}$ and $\bar{\varphi}=\varphi_{L V}=\varphi_{V L}($ see Theorem 5.12).

(a) Then $\bar{S} \subset \mathfrak{P}$. Consequently, if $f$ is essentially separably valued and in $\bar{S}$, then $f \in \mathfrak{B}$. In particular, $S_{L} \subset \mathfrak{B}$.

(b) Suppose there exists an $\alpha \in E_{c}^{\sim+}$ with the property that if $b \in E$ and $b>0$, then $\alpha(b)>0$. Then $\mathfrak{B}_{V} \subset \mathfrak{B}$. Consequently, $\bar{S} \subset \mathfrak{B}$.

Proof (a) Because $\|\cdot\|$ is $\sigma$-order continuous, $E^{\prime}=E_{c}^{\sim}$. Therefore Theorem 8.14 implies that $\bar{S} \subset \mathfrak{P}$.

Note that $S_{L} \subset \mathfrak{B}$. Since $\mathfrak{B}$ is a Riesz ideal in the space of strongly measurable functions $X \rightarrow E$, an $f \in \bar{S}$ is an element of $\mathfrak{B}$ if it is essentially separably valued, since there are elements $\sigma, \tau \in S_{L}$ with $\sigma \leq f \leq \tau$ and $f$ is weakly measurable since $f \in \mathfrak{P}$.

(b) Suppose $f \in \mathfrak{B}_{V}$ and $\sigma_{n}, \tau_{n} \in \mathfrak{B}$ are such that $\sigma_{n} \leq f \leq \tau_{n}$ for $n \in \mathbb{N}, \sigma_{n} \uparrow, \tau_{n} \downarrow$ and $\sup _{n \in \mathbb{N}} \mathfrak{b}\left(\sigma_{n}\right)=\mathfrak{b}_{V}(f)=\inf _{n \in \mathbb{N}} \mathfrak{b}\left(\tau_{n}\right)$. Then $\inf _{n \in \mathbb{N}} \int \alpha \circ\left(\tau_{n}-\sigma_{n}\right) \mathrm{d} \mu=$ $\alpha\left(\inf _{n \in \mathbb{N}} \mathfrak{b}\left(\tau_{n}-\sigma_{n}\right)\right)=0$ and therefore $\alpha\left(\inf _{n \in \mathbb{N}}\left(\tau_{n}-\sigma_{n}\right)\right)=\inf _{n \in \mathbb{N}} \alpha \circ\left(\tau_{n}-\sigma_{n}\right)$ is integrable with integral equal to zero. Therefore $\inf _{n \in \mathbb{N}}\left(\tau_{n}-\sigma_{n}\right)=0$ a.e., hence $\tau_{n} \rightarrow f$ a.e. Therefore $f$ is strongly measurable and thus $f \in \mathfrak{B}$ by (a). By (a) $S_{L} \subset \mathfrak{B}$, hence $\bar{S}=S_{L V} \subset \mathfrak{B}$.

9.6 For the next theorem we write $S_{\mathbb{R}}$ for the space of simple functions $X \rightarrow \mathbb{R}$. Note that if $u \in E^{+}$and $\pi$ is an element of $\left(S_{\mathbb{R}}\right)_{L}^{+}$, thus of $\mathcal{L}^{1}(\mu)$, then $u \pi \in S_{L}$ and $\varphi_{L}(u \pi)=u \int \pi \mathrm{d} \mu$. 
Theorem 9.7 Let $Y$ be a compact Hausdorff space and let $E=C(Y)$ be equipped with the supremum norm, $\|\cdot\|_{\infty}$. Then $\mathfrak{B} \subset S_{L V}$ and $\varphi_{L V}=\mathfrak{b}$ on $\mathfrak{B}$.

Proof Let $f \in \mathfrak{B}$ and $\left(s_{n}\right)_{n \in \mathbb{N}}$ be a sequence of simple functions $X \rightarrow E$ such that $\int\left\|f-s_{n}\right\|_{\infty} \mathrm{d} \mu \leq \frac{1}{n 2^{n}}$ for all $n$. Then there exists an integrable function $g: X \rightarrow$ $[0, \infty)$ with $g \geq \sum_{n \in \mathbb{N}} n\left\|f-s_{n}\right\|_{\infty} \mu$-a.e., and thus $g \geq n\left\|f-s_{n}\right\|_{\infty} \mu$-a.e. By Theorem 7.1 there exists a $\pi \in\left(S_{\mathbb{R}}\right)_{L}^{+}$(see 9.6) with $\pi \geq g$. Then, let $Z \in \mathcal{A}$ with $\mu(A)=0$ be such that $\left\|f(x)-s_{n}(x)\right\|_{\infty} \leq \frac{1}{n} \pi(x)$ for all $x \in X \backslash Z$. We may, by replacing $s_{n}$ by $s_{n} \mathbb{1}_{X \backslash Z}$, assume that $s_{n}=0$ on $Z$. With $Z_{k}=\{x \in Z: k-1<$ $\left.\|f(x)\|_{\infty} \leq k\right\}$ and $\rho$ the element in $S_{L}^{+}$with $\rho(x)=0$ for $x \notin Z$ and $\rho(x)=k \mathbb{1}_{Y}$ for $x \in Z_{k}$, we have

$$
s_{n}-\frac{1}{n} \mathbb{1}_{Y} \pi-\rho \leq f \leq s_{n}+\frac{1}{n} \mathbb{1}_{Y} \pi+\rho \quad(n \in \mathbb{N}) .
$$

Because $\varphi_{L}(\rho)=0, \varphi_{L}\left(\frac{1}{n} \mathbb{1}_{Y} \pi\right) \rightarrow 0, \varphi_{L}\left(s_{n}\right)=\mathfrak{b}\left(s_{n}\right)$ and $\mathfrak{b}\left(s_{n}\right) \rightarrow \mathfrak{b}(f)$, both $\varphi_{L}\left(s_{n}-\frac{1}{n} \mathbb{1}_{Y} \pi-\rho\right)$ and $\varphi_{L}\left(s_{n}+\frac{1}{n} \mathbb{1}_{Y} \pi+\rho\right)$ converge to $\mathfrak{b}(f)$. Whence

$$
\mathfrak{b}(f)=\sup _{n \in \mathbb{N}} \varphi_{L}\left(s_{n}-\frac{1}{n} \mathbb{1}_{Y} \pi-\rho\right)=\inf _{n \in \mathbb{N}} \varphi_{L}\left(s_{n}+\frac{1}{n} \mathbb{1}_{Y} \pi+\rho\right) .
$$

Thus $f \in S_{L V}$ and $\varphi_{L V}(f)=\mathfrak{b}(f)$.

By the Yosida Representation Theorem the following is an immediate consequence.

Corollary 9.8 Let E be a Archimedean Riesz space with strong unit u and assume $E$ is uniformly complete, i.e., $E$ is a Banach lattice under the norm $\|\cdot\|_{u}$ given by $\|x\|_{u}=\inf \{\lambda \in[0, \infty):|x| \leq \lambda u\}$. Then $\mathfrak{B} \subset S_{L V}$ and $\varphi_{L V}=\mathfrak{b}$ on $\mathfrak{B}$.

Example 9.9 (I) Take $X=\mathbb{N}, \mathcal{A}=\mathcal{P}(\mathbb{N})$, and let $\mu$ be the counting measure. We have $S=c_{00}[E] ; S_{V}=c_{00}[E]$; all functions $\mathbb{N} \rightarrow E$ are partially in $S ; \bar{S}:=$ $S_{L V}=S_{V L}=S_{L}$ (see Theorem 5.5(c)) and $\bar{S}^{+}$consists precisely of the functions $f: \mathbb{N} \rightarrow E^{+}$for which $\sum_{n} f(n)$ exists in the sense of the ordering. On the other hand, $f: \mathbb{N} \rightarrow E$ is Bochner integrable if and only if $\sum_{n=1}^{\infty}\|f(n)\|<\infty$.

- If $\|\cdot\|$ is a $\sigma$-order continuous norm, then $\mathfrak{B} \subset \bar{S}$.

- Moreover $\|\cdot\|$ is equivalent to an abstract L-norm if and only if $\mathfrak{B}=\bar{S}$ (since, if $\mathfrak{B}=\bar{S}$, the following holds: if $x_{1}, x_{2}, \ldots \in E^{+}$and $\sum_{n} x_{n}$ exists, then $\sum_{n \in \mathbb{N}}\left\|x_{n}\right\|<\infty$, see Theorem 12.1).

- For $E=c_{0}$ there exists an $f \in \mathfrak{P}$ that is not in $\bar{S}$. For example $f: \mathbb{N} \rightarrow c_{0}$ given by

$$
f=\left(e_{1},-e_{1}, e_{2},-e_{2}, e_{3},-e_{3}, \ldots\right)
$$

is Pettis integrable since $c_{0}^{\prime} \cong \ell^{1}$ has basis $\left\{\delta_{n}: n \in \mathbb{N}\right\}$ where $\delta_{n}(x)=x(n)$ and $\sum_{m \in \mathbb{N}} \delta_{n}(f(m))=0$ for all $m \in \mathbb{N}$. $c_{0}$ is $\sigma$-Dedekind complete and thus by Theorem 4.32 the set $\bar{S}$ is a Riesz space. However, $|f|$ is not in $\bar{S}$ and therefore neither $f$ is. 
- For $E=c$ there exists an $f \in \bar{S}$ that is not in $\mathfrak{B}$ and not in $\mathfrak{P}$ : Consider for example $f: n \mapsto e_{n}$. It is an element of $\bar{S}$ but not of $\mathfrak{B}$. It is not even Pettis integrable. (Suppose it is, and its integral is $a$. Then for all $u \in c^{\prime}$ we have $u(a)=\int u \circ f \mathrm{~d} \mu=\sum_{n=1}^{\infty} u(f(n))=\sum_{n=1}^{\infty} u\left(e_{n}\right)$. Letting $u$ be the coordinate functions, we see that $a(n)=1$ for all $n \in \mathbb{N}$; letting $u$ be $x \mapsto \lim _{n \rightarrow \infty} x(n)$ we have a contradiction.)

(II) $\mathfrak{B} \not \subset S_{V L V}$. Let $(\mathbb{R}, \mathcal{M}, \lambda)$ be the Lebesgue measure space. Let $E$ be the $\sigma$ Dedekind complete Riesz space $L^{1}(\lambda)$. Let $g \in L^{1}(\lambda)$ be the equivalence class of the function that equals $t^{-\frac{1}{2}}$ for $0<t \leq 1$ and equals 0 for other $t$. Let $L_{x} g(t)=g(t-x)$ for $x \in \mathbb{R}$. Then the function $f: \mathbb{R} \rightarrow L^{1}(\lambda)$ for which $f(x)=\mathbb{1}_{[0,1]}(x) L_{x} g$ is Bochner integrable $\left(f\right.$ is continuous in the $\|\cdot\|_{1}$ norm (because $\left\|L_{\varepsilon} g-g\right\|_{1}=2 \sqrt{\varepsilon}$ for $\left.\varepsilon>0\right)$ and $\int\|f(x)\|_{1} \mathrm{~d} \lambda(x)=\iint \mid g(t-$ $\left.x) \mid \mathrm{d} \lambda(t) \mathrm{d} \lambda(x)=\|g\|_{1}<\infty\right)$ but no element of $S_{V L V}$ (by Theorem 8.3).

\section{Extensions of Bochner integrable functions}

\section{Consider the situation of Sect. 9 .}

As we have seen in Examples 9.9, e.g., (95), the set of Pettis integrable functions need not be stable. We show that $\mathfrak{B}$ is stable and $\mathfrak{b}$ is laterally extendable. Furthermore we give an example of an $f \in \mathfrak{B}_{L V}$ that is neither in $S_{V L V}$, nor in $\mathfrak{B}_{L}$ or $\mathfrak{B}_{V}$.

Theorem $10.1 \mathfrak{B}$ is stable and $\mathfrak{b}$ is laterally extendable.

Proof Note that $f \mathbb{1}_{B} \in \mathfrak{B}$ for all $f \in \mathfrak{B}$ and $B \in \mathcal{A}$ (since $f \mathbb{1}_{B}$ is strongly measurable and $\left\|f \mathbb{1}_{B}\right\|$ is integrable), i.e., $\mathfrak{B}$ is stable. Let $\left(A_{n}\right)_{n \in \mathbb{N}}$ be a partition in $\mathcal{A}$ of $X$. Let $f: X \rightarrow E^{+}$be a Bochner integrable function. Then $\int\|f\| \mathrm{d} \mu<\infty$ and with $B_{n}=A_{1} \cup \cdots \cup A_{n}$ and Lebesgue's Dominated Convergence Theorem we obtain

$$
\left\|\mathfrak{b}\left(f-\sum_{n=1}^{N} f \mathbb{1}_{A_{n}}\right)\right\| \leq \int\left\|f(x)-\mathbb{1}_{B_{N}}(x) f(x)\right\| \mathrm{d} \mu(x) \rightarrow 0 .
$$

Thus

$$
\mathfrak{b}(f)=\lim _{N \rightarrow \infty} \sum_{n=1}^{N} \mathfrak{b}\left(f \mathbb{1}_{A_{n}}\right)=\sum_{n} \mathfrak{b}\left(f \mathbb{1}_{A_{n}}\right)
$$

We conclude that $\mathfrak{b}$ is laterally extendable.

In the following situation we have $\mathfrak{B}_{L}=\mathfrak{B}=\mathfrak{B}_{V}$.

Lemma 10.2 Let $E$ be a Banach lattice with an abstract L-norm (i.e., $\|a+b\|=$ $\|a\|+\|b\|$ for $\left.a, b \in E^{+}\right)$.

(a) Then

$$
\|\mathfrak{b}(f)\|=\int\|f\| \mathrm{d} \mu \quad\left(f \in \mathfrak{B}^{+}\right)
$$


(b) $\mathfrak{B}_{L}=\mathfrak{B}$.

(c) There exist an $\alpha \in E_{c}^{\sim+}$ as in Theorem 9.5(b). Consequently $\mathfrak{B}_{V}=\mathfrak{B}$.

Proof (a) It is clear that $\|\mathfrak{b}(f)\|=\int\|f\| \mathrm{d} \mu$ for $f \in S^{+}$, hence by limits for all $f \in \mathfrak{B}^{+}$.

(b) Suppose $f \in \mathfrak{B}_{L}^{+}$. Let $\left(A_{n}\right)_{n \in \mathbb{N}}$ be a $\mathfrak{b}$-partition for $f$, write $f_{n}=f \mathbb{1}_{A_{n}}$. Then $\left\|\sum_{n=1}^{N} f_{n}-f\right\| \rightarrow 0$, hence $f$ is strongly measurable. Moreover, since $\|\cdot\|$ is $\sigma$ order continuous $\left\|\sum_{n=1}^{N} \mathfrak{b}\left(f_{n}\right)-\mathfrak{b}_{L}(f)\right\| \rightarrow 0$, hence $\sum_{n=1}^{N}\left\|\mathfrak{b}\left(f_{n}\right)\right\| \rightarrow \mathfrak{b}_{L}(f)$. Using (a) we obtain $\int\|f\| \mathrm{d} \mu=\sum_{n \in \mathbb{N}} \int\left\|f_{n}\right\| \mathrm{d} \mu=\sum_{n \in \mathbb{N}}\left\|\mathfrak{b}\left(f_{n}\right)\right\|<\infty$, i.e., $f \in \mathfrak{B}$.

(c) Extend $\alpha: E^{+} \rightarrow \mathbb{R}$ given by $\alpha(b)=\|b\|$ to a linear map on $E$.

10.3 Consider the situation of Example 8.8. Since $S \subset \mathfrak{B}$ and $\varphi(h)=\mathfrak{b}(h)$ for $h \in S: f \in \mathfrak{B}_{V}$. The function $f$ is not essentially separably-valued (i.e., $f(X \backslash A)$ is not separable for all null sets $A \in \mathcal{A}$ ), hence $f$ (and thus $g$ ) is not strongly measurable (see [3, Theorem3.5.2]). Hence $f$ is not Bochner integrable, i.e., $f \in \mathfrak{B}_{V}$ but $f \notin \mathfrak{B}$.

In a similar way as has been shown in Example 8.8, one can show that $g: \mathbb{R} \rightarrow E^{+}$ defined by $g(t)=\mathbb{1}_{\{t\}}$ for $t \in \mathbb{R}$ is in $S_{L V}$. Then $g \in \mathfrak{B}_{L V}$ but $g \notin \mathfrak{B}_{V}$.

10.4 All $f \in \mathfrak{B}_{L}$ are strongly measurable. Therefore for $f \in \mathfrak{B}_{L}$ we have $f \notin \mathfrak{B}$ if and only if $\int\|f\| \mathrm{d} \mu=\infty$.

The following example illustrates that by extending the Bochner integrable functions one can obtain more than by extending the simple functions.

Example $10.5\left[\psi \in \mathfrak{B}_{V}, \psi \notin \mathfrak{B}\right]$

Let $X=[2,3]$, let $\mathcal{A}$ be the set of Lebesgue measurable subsets of $X$ and $\mu$ be the Lebesgue measure on $X$. Let $M$ denote the set of equivalence classes of measurable functions $\mathbb{R} \rightarrow \mathbb{R}$. Let

$E=\left\{f \in M: \sup _{x \in \mathbb{R}} \int_{x}^{x+1}|f|<\infty\right\}, \quad\|\cdot\|: E \rightarrow[0, \infty), \quad\|f\|=\sup _{x \in \mathbb{R}} \int_{x}^{x+1}|f|$.

Then $E$ equipped with the norm $\|\cdot\|$ is a Banach lattice. $E$ is an ideal in $M$ and therefore $\sigma$-Dedekind complete (hence $S_{V}$ is stable; 4.25). The norm $\|\cdot\|$ is not $\sigma$-order continuous.

For $a \in \mathbb{R}, c>0$ define $S_{a, c}: X \rightarrow E^{+}$by $S_{a, c}(x)=\mathbb{1}_{(a+c x, \infty)}$. If $x, y \in X$ with $y>x$ then $\left\|S_{a, c}(x)-S_{a, c}(y)\right\| \leq\left\|\mathbb{1}_{(a+c x, a+c y]}\right\| \leq c|x-y|$, so $S_{a, c}$ is continuous and therefore strongly measurable. Furthermore $\left\|S_{a, c}(x)\right\|=1$ for all $x \in X$, i.e., $x \mapsto\left\|S_{a, c}(x)\right\|$ is integrable. Thus $S_{a, c}$ is Bochner integrable. For $d, e \in \mathbb{R}$ with $e>d$ the map $E \rightarrow \mathbb{R}, f \mapsto \int_{d}^{e} f$ is a continuous linear functional. Therefore

$$
\int_{d}^{e} \mathfrak{b}\left(S_{a, c}\right)=\int_{X} \int_{d}^{e}\left(S_{a, c}(x)\right)(t) \mathrm{d} t \mathrm{~d} x=\int_{d}^{e} \int_{X}\left(S_{a, c}(x)\right)(t) \mathrm{d} x \mathrm{~d} t .
$$


Since this holds for all $d, e \in \mathbb{R}$ with $e>d$, for $t \in \mathbb{R}$ we have

$$
\left(\mathfrak{b}\left(S_{a, c}\right)\right)(t)=\int_{X}\left(S_{a, c}(x)\right)(t) \mathrm{d} x=\int_{2}^{3} \mathbb{1}_{(a+c x, \infty)}(t) \mathrm{d} x=\left(\frac{t-a}{c} \wedge 3-2\right) \vee 0 .
$$

For $k \in \mathbb{N}$ define $r_{k}, R_{k}: X \rightarrow E$ by

$$
R_{k}:=S_{0, k}, \quad r_{k}:=S_{0, k}-S_{1, k}
$$

For $x \in X$ and $k \in \mathbb{N}, r_{k}(x)=\mathbb{1}_{(k x, k x+1]}$ and $k x+1<(k+1) x$. Define

$$
\psi(x):=\mathbb{1}_{\bigcup_{k \in \mathbb{N}}(k x, k x+1]}=\sum_{k \in \mathbb{N}} r_{k}(x), \quad \sigma_{n}:=\sum_{k=1}^{n} r_{k}, \quad \tau_{n}:=\sum_{k=1}^{n} r_{k}+R_{n+1} .
$$

Note that $\sigma_{n} \leq \psi \leq \tau_{n}$ and $\sigma_{n}, \tau_{n} \in \mathfrak{B}$ all for $n \in \mathbb{N}$. Since $E$ is $\sigma$-Dedekind complete and therefore mediated, from the fact that

$$
\inf _{n \in \mathbb{N}} \mathfrak{b}\left(\tau_{n}-\sigma_{n}\right)=\inf _{n \in \mathbb{N}} \mathfrak{b}\left(R_{n+1}\right)=0,
$$

it follows that $\psi \in \mathfrak{B}_{V}$. However, $\psi \notin \mathfrak{B}$ since $\psi$ is not essentially separably valued:

Let $x, y \in X, x<y$. We prove $\|\psi(x)-\psi(y)\| \geq 1$. For $k \in \mathbb{N}$ :

$$
\begin{aligned}
k-1 \leq \frac{1}{y-x}<k & \Longrightarrow\left\{\begin{array}{l}
1+(k-1) y \leq k x, \\
1+k x<k y,
\end{array}\right. \\
& \Longrightarrow(k x, k x+1] \cap \bigcup_{i \in \mathbb{N}}(i y, i y+1]=\emptyset .
\end{aligned}
$$

Hence $\|\psi(x)-\psi(y)\| \geq 1$ for all $x, y \in X$ with $x \neq y$.

So $\psi$ is an element of $\mathfrak{B}_{V}$ but not of $\mathfrak{B}$ (and neither of $\mathfrak{B}_{L}$ ).

Example 10.6 $\left[f \in \mathfrak{B}_{L V}, f \notin \mathfrak{B}_{L}, f \notin \mathfrak{B}_{V}, f \notin S_{V L V}\right]$

Let $(X, \mathcal{A}, \mu)$ be the Lebesgue measure space $(\mathbb{R}, \mathcal{M}, \lambda)$. Let $E$ and $\psi$ be as in Example 10.5. Define $u: \mathbb{R} \rightarrow E$ by

$$
u(x)= \begin{cases}\psi(x) & x \in[2,3] \\ 0 & \text { otherwise }\end{cases}
$$

Then $u$ is an element of $\mathfrak{B}_{V}$ and not of $\mathfrak{B}_{L}$. As we have seen in Examples 9.9(II) there exists a $g$ in $L^{1}(\lambda)$ and thus in $E$ such that $v: x \mapsto \mathbb{1}_{[0,1]}(x) L_{x} g$ is an element of $\mathfrak{B}$ that is not an element of $S_{V L V}$. Furthermore $w: \mathbb{R} \rightarrow E$ given by $w(x)=\mathbb{1}_{(n, n+1]}$ for $x \in(n, n+1]$ is an element of $\mathfrak{B}_{L}$ and not of $\mathfrak{B}_{V}$. Therefore $f=u+v+w$ is an element of $\mathfrak{B}_{L V}$ (and thus of $\mathfrak{B}_{V L}$; see Theorem 5.8) but is neither an element of $S_{V L V}$ nor of $\mathfrak{B}_{V}$ or $\mathfrak{B}_{L}$. 


\section{Discussion}

Of course, to some extent our approach is arbitrary. We mention some alternatives, with comments.

11.1 The reader may have wondered why in our definition of the lateral extension the sets $A_{n}$ are required not only to be disjoint but also to cover $X$ (i.e., to form a partition). Without the covering of $X$ the definition remains perfectly meaningful, but the sum of two positive laterally integrable functions need not be laterally integrable, even in quite natural situations. (E.g., take $E=F=\mathbb{R}$ and $X=[0,1]$; let $\mathcal{I}$ be the ring generated by the open intervals, $\Gamma$ the space of all Riemann integrable functions on $[0,1]$, and $\varphi$ the Riemann integral. If $f$ is the indicator of the Cantor set, then $\mathbb{1}-f$ is laterally integrable but $2 \mathbb{1}-f$ is not.)

11.2 For the vertical extension we have, somewhat artificially, introduced a countability restriction leading us from $\varphi_{v}$ to $\varphi_{V}$; see Definition 3.3. In some sense, $\varphi_{v}$ would have served as well as $\varphi_{V}$. In order to get a non-void theory, however, we would need a much stronger (but analogous) condition than "mediatedness", restricting our world drastically.

11.3 A different approach to both the vertical and the lateral extension, closer to Daniell and Bourbaki, could run as follows. Starting from the situation of 3.14, call a function $X \rightarrow F^{+}$"integrable" if there exist $f_{1}, f_{2}, \ldots \in \Gamma^{+}$such that

$$
\left\{\begin{array}{l}
f_{n} \uparrow f \text { in } F^{X}, \\
\sup _{n \in \mathbb{N}} \varphi\left(f_{n}\right) \text { exists in } E,
\end{array}\right.
$$

then define the "integral" $\bar{\varphi}(f)$ of $f$ by

$$
\bar{\varphi}(f):=\sup _{n \in \mathbb{N}} \varphi\left(f_{n}\right) .
$$

This definition is meaningful only if, in the above situation

$$
g \in \Gamma^{+}, g \leq f, \Longrightarrow \varphi(g) \leq \sup _{n \in \mathbb{N}} \varphi\left(f_{n}\right)
$$

which in a natural way leads to the requirement that $\Gamma$ be a lattice and that $\varphi$ be continuous in the following sense:

$$
h_{1}, h_{2}, \ldots \in \Gamma^{+}, h_{n} \downarrow 0 \Longrightarrow \varphi\left(h_{n}\right) \downarrow 0 .
$$

These conditions lead to a sensible theory, but again we consider them as too restrictive. (See Example II.2.4 in the thesis of Jeurnink [16] for an example of a $\Gamma$ that consists of simple functions on a measure space with values in a $C(X)$ for which (110) does not hold for the standard integral on simple functions (see 4.33).) 
Acknowledgments The authors are grateful to O. van Gaans for valuable discussions. W.B. van Zuijlen is supported by ERC Advanced Grant VARIS-267356.

Open Access This article is distributed under the terms of the Creative Commons Attribution 4.0 International License (http://creativecommons.org/licenses/by/4.0/), which permits unrestricted use, distribution, and reproduction in any medium, provided you give appropriate credit to the original author(s) and the source, provide a link to the Creative Commons license, and indicate if changes were made.

\section{Appendix}

Theorem 12.1 Let $E$ be a Banach lattice with the property

$$
\text { If } x_{1}, x_{2}, \ldots \in E^{+} \text {and } \sum_{n} x_{n} \text { exists, then } \sum_{n \in \mathbb{N}}\left\|x_{n}\right\|<\infty \text {. }
$$

Then the norm $\|\cdot\|$ is equivalent to an L-norm.

The proof uses the following lemma.

Lemma 12.2 Let $E$ be a Banach lattice that satisfies (12.1). Then there exists a $C>0$ such that

$$
x_{1}, x_{2}, \ldots \in E^{+}, \sum_{n} x_{n} \text { exists } \Longrightarrow \sum_{n \in \mathbb{N}}\left\|x_{n}\right\| \leq C\left\|\sum_{n} x_{n}\right\| .
$$

Proof Suppose not. For $i \in \mathbb{N}$ let $x_{i 1}, x_{i 2}, \ldots \in E^{+}, \sum_{n} x_{i n}=b_{i}$ and $\sum_{n \in \mathbb{N}}\left\|x_{i n}\right\|>$ $2^{i}\left\|b_{i}\right\|$ and $\left\|b_{i}\right\|=2^{-i}$. Then $\sum_{i \in \mathbb{N}}\left\|b_{i}\right\|<\infty$, so $\sum_{i} b_{i}$ exists. As $\sum_{i} b_{i}=$ $\sum_{i} \sum_{n} x_{i n}$, by (12.1) we get $\infty>\sum_{i \in \mathbb{N}} \sum_{n \in \mathbb{N}}\left\|x_{i n}\right\|>\sum_{i \in \mathbb{N}} 2^{i}\left\|b_{i}\right\|=\infty$.

Proof of Theorem 12.1 By Lemma 12.2 we can define $p: E \rightarrow[0, \infty)$,

$$
p(x)=\sup \left\{\sum_{n \in \mathbb{N}}\left\|x_{n}\right\|: x_{1}, x_{2}, \ldots \in E^{+}, \sum_{n} x_{n} \leq|x|\right\},
$$

obtaining $p(x)=p(|x|), p(t x)=|t| p(x),\|x\| \leq p(x) \leq C\|x\|$ for all $x \in E, t \in \mathbb{R}$ (with $C$ as in Lemma 12.2) and $p(x) \leq p(y)$ for $x, y \in E^{+}$with $x \leq y$.

Let $x, y \in E^{+}$; we prove $p(x+y)=p(x)+p(y)$.

- For $\varepsilon>0$ choose $x_{1}, x_{2}, \ldots, y_{1}, y_{2}, \ldots \in E^{+}, \sum_{n} x_{n} \leq x, \sum_{n} y_{n} \leq y$, $\sum_{n \in \mathbb{N}}\left\|x_{n}\right\| \geq p(x)-\varepsilon, \sum_{n \in \mathbb{N}}\left\|y_{n}\right\| \geq p(y)-\varepsilon$. Considering the sequence $x_{1}, y_{1}, x_{2}, y_{2}, \ldots$ we find $\sum_{n \in \mathbb{N}}\left(\left\|x_{n}\right\|+\left\|y_{n}\right\|\right) \leq p(x+y)$. Hence $p(x+y) \geq$ $p(x)+p(y)$.

- On the other hand: Let $z_{1}, z_{2}, \ldots \in E^{+}, \sum_{n} z_{n} \leq x+y$; we prove $\sum_{n \in \mathbb{N}}\left\|z_{n}\right\| \leq$ $p(x)+p(y)$. Define $u_{n}, v_{n}$ by

$$
u_{1}+\cdots+u_{n}=\left(z_{1}+\cdots+z_{n}\right) \wedge x, \quad v_{n}=z_{n}-u_{n} \quad(n \in \mathbb{N}) .
$$


Then $\left(z_{1}+\cdots+z_{n}\right) \wedge x-z_{n}=\left(z_{1}+\cdots+z_{n}-z_{n}\right) \wedge\left(x-z_{n}\right) \leq\left(z_{1}+\cdots+z_{n-1}\right) \wedge x$, implying $u_{n}-z_{n} \leq 0$; and $\left(z_{1}+\cdots+z_{n}\right) \wedge x \geq\left(z_{1}+\cdots+z_{n-1}\right) \wedge x$, implying $u_{n} \geq 0$. Thus

$$
u_{n} \geq 0, v_{n} \geq 0 \quad(n \in \mathbb{N})
$$

$\sum_{n \in \mathbb{N}}\left\|u_{n}\right\| \leq \sum_{n \in \mathbb{N}}\left\|z_{n}\right\|<\infty$, so $\sum_{n} u_{n}$ exists; $\sum_{n} u_{n} \leq x$, and $\sum_{n \in \mathbb{N}}\left\|u_{n}\right\| \leq$ $p(x) . \sum_{n \in \mathbb{N}}\left\|v_{n}\right\| \leq \sum_{n \in \mathbb{N}}\left\|z_{n}\right\|<\infty$, so $\sum_{n} v_{n}$ exists. For every $n \in \mathbb{N}, z_{1}+$ $\cdots+z_{n} \leq\left(z_{1}+\cdots+z_{n}+y\right) \wedge(x+y)=\left(z_{1}+\cdots+z_{n}\right) \wedge x+y=u_{1}+\cdots+u_{n}+y$, so $v_{1}+\cdots+v_{n} \leq y$; then $\sum_{n} v_{n} \leq y$ and $\sum_{n \in \mathbb{N}}\left\|v_{n}\right\| \leq p(y)$.

Thus $\sum_{n \in \mathbb{N}}\left\|z_{n}\right\| \leq \sum_{n \in \mathbb{N}}\left\|u_{n}\right\|+\sum_{n \in \mathbb{N}}\left\|v_{n}\right\| \leq p(x)+p(y)$.

\section{References}

1. Bochner, S.: Integration von Funktionen, deren Werte die Elemente eines Vektorraumes sind. Fund. Math. 20, 262-276 (1933)

2. Pettis, B.J.: On integration in vector spaces. Trans. Am. Math. Soc. 44, 277-304 (1938)

3. Hille, E., Phillips, R.S.: Functional Analysis and Semi-Groups, vol. 31. American Mathematical Society, USA (1957)

4. Daniell, P.J.: A general form of integral. Ann. Math. Sec. Ser. 19, 279-294 (1918)

5. Bourbaki, N.: Livre VI Intégration. Hermann, Mainz (1952)

6. de Pagter, B.: On z-ideals and d-ideals in Riesz spaces III. Indag. Math. 43, 409-422 (1981)

7. Birkhoff, G.: Lattice Theory, vol. XXV, 3rd ed. American Mathematical Society, USA (1967)

8. Anthony, L.: Peressini Publishers, Ordered topological vector spaces. Harper \& Row, New York (1967)

9. Bonsall, F.F.: Sublinear functionals and ideals in partially ordered vector spaces. Proc. Lond. Math. Soc. 4(3), 402-418 (1954)

10. Fuchs, L.: Riesz vector spaces and Riesz algebras. In: Séminaire Dubreil. Algèbre et théorie des nombres, vol. 19, no. 23-24, pp 1-9 (1965-1966)

11. van Gaans, O., Kalauch, A.: Ideals and bands in pre-Riesz spaces. Positivity 12, 591-611 (2008)

12. van Gaans, O., Kalauch, A.: Bands in pervasive pre-Riesz spaces. Oper. Matr. 2(2), 177-191 (2008)

13. Zaanen, A.C.: Riesz Spaces II. North-Holland Publishing Company, Amsterdam (1983)

14. Semadeni, Zbigniew: Banach Spaces of Continuous Functions, vol. 1. PWN Polish Scientific Publishers, Warszawa (1971)

15. de Jonge, E., van Rooij, A.C.M.: Introduction to Riesz Spaces. Mathematisch Centrum, Amsterdam (1977)

16. Jeurnink, G.A.: Integration of functions with values in a Banach lattice. $\mathrm{PhD}$ thesis, University of Nijmegen (1982) 